\title{
Hybrid graphene/geopolymeric cement as a superionic conductor for structural health monitoring applications
}

M.Saafi*, G. Piukovics and J. Ye

Department of Engineering

Lancaster University

Gillow Avenue

Lancaster, LA1 4YW, UK

*m.saafi@lancaster.ac.uk

\begin{abstract}
In this paper, we demonstrate for the first time a novel hybrid superionic long gauge sensor for structural health monitoring applications. The sensor consists of two graphene electrodes and a superionic conductor film made entirely of fly ash geopolymeric material. The sensor employs ion hopping as a conduction mechanism for high precision temperature and tensile strain sensing in structures. The design, fabrication and characterization of the sensor are presented. The temperature and strain sensing mechanisms of the sensor are also discussed. The experimental results revealed that the crystal structure of the superionic film is a 3D sodium-poly(sialate-siloxo) (Na-PSS) framework, with a room temperature ionic conductivity between $1.54 \times 10^{-2}$ and $1.72 \times 10^{-2} \mathrm{~S} / \mathrm{m}$ and, activation energy of $0.156 \mathrm{eV}$, which supports the notion that ion hopping is the main conduction mechanism for the sensor. The sensor showed high sensitivity to both temperature and tensile strain. The sensor exhibited temperature sensitivity as high as $21.5 \mathrm{k} \Omega /{ }^{\circ} \mathrm{C}$ and tensile strain sensitivity (i.e., gauge factor) as high as 358. The proposed sensor is relatively inexpensive and can easily be manufactured with long gauges to measure temperature and bulk strains in structures. With some further development and characterization, the sensor can be retrofitted onto existing structures such as bridges, buildings, pipelines and wind turbines to monitor their structural integrity.
\end{abstract}

\section{Introduction}

Structural health monitoring (SHM) has the potential to improve the way engineering structures are monitored and inspected for enhanced structural safety and sustainability. 
However, current SHM approaches still have some limitations for widespread deployment in large structures due to several issues associated with the current sensing technologies, including high costs, incompatibility with the host structures, deterioration in aggressive environments and, expensive and complex signal processing algorithms [1]. Recent advances in functional materials offer tremendous promise for low-cost, highly sensitive and durable sensors that could potentially improve the cost-effectiveness and robustness of SHM techniques. For example, piezoresistive strain sensors based on conductive composites have been successfully developed for several SHM applications. These sensors are typically in the form of a polymer matrix with conductive fillers such as carbon fibers (CFs), carbon nanotubes/nanofibers (CNTs/CNFs) and graphene [2-5]. Their sensing mechanism is based on the change in the electrical properties in response to mechanical deformation. Because polymer-based sensors are inherently vulnerable to damage in harsh environment applications such as bridges, tunnels and wind turbines, researchers have developed self-sensing cementitious materials through the addition of CFs and CNTs/CNFs conductive fillers [6-8], as an alternative. Engineered self-sensing cementitious materials offer several advantages including low-costs, chemical stability, good durability in demanding environments, good compatibility with host structures and ease of fabrication and interrogation [9]. Although conductive composites-based piezoresistive sensors are regarded as a mature sensing technology, significant technical problems are still holding back their full potential in SHM applications. One of the biggest challenges in synthesizing these composite sensors is the dispersion of CFs, CNTs/CNFs and graphene conductive fillers. These fillers tend to agglomerate and create defects, thus exhibiting a poor piezoresistive response, particularly to small strains. The high cost of these fillers is another challenge as it renders the sensors financially unattractive for large-scale deployment.

Recently, there has been a growing interest in developing cost-effective superionic conductors (SICs) for a number of applications including semiconductor devices, fuel cells 
and solid-state batteries. SICs include among others lithium and sodium-based solid state conductors [10-12]. Depending on the doping and processing conditions, the electrical conductivity of SICs can be in the range of $10^{-3}-12 \mathrm{mS} / \mathrm{cm}$ [10-12]. Unlike composites with conductive fillers, the conduction mechanism in SICs relies on the intrinsic and extrinsic defects (also known as electron holes) in the crystalline framework to conduct current through ion hopping [13]. The electrical conductivity of SICs depends partially on the ion diffusion mechanism and the concentration of mobile ions [13]. SICs-based sensors have yet to be developed for SHM applications; however, their piezoresistive behavior is to some extent similar to that of semiconductors, where the change in the electrical conductivity in response to mechanical strain is attributed to the change in the electronic bandgap structure and hopping sites resulting from the change in the inter-atomic spacing [14]. On the other hand, the change in the electrical conductivity of conductive composites in response to mechanical strain is mainly due to the dimensional change when strained. As a result, the room temperature gauge factor (GF) of semiconductors is significantly higher than that of conductive composites, thereby allowing small strains to be measured. This is particularly important as crack propagation in brittle materials such as concrete typically occurs at low tensile strain levels. Because of their unique properties, SICs could lead to a new generation of low-cost and highly sensitive sensors for SHM applications.

In this paper, a novel low-cost and long gauge SIC sensor for temperature and tensile strain sensing is presented. The sensor consists of two graphene electrodes and a sodiumpoly(sialate-siloxo) (Na-PSS) geopolymeric film manufactured entirely from fly ash with a tuned crystal structure to exhibit a superionic behavior with giant strain and temperature sensitivity coefficients. To the authors' knowledge, this is the first time a graphene/Na-PSSbased SIC sensor has been proposed for SHM applications.

\section{Experimental program}

\section{1. Sensor design, materials and chemicals}


As shown in Fig. 1, the sensor consists of two coplanar electrodes embedded into a SIC sensing element with a gauge length of $60 \mathrm{~mm}$ and thickness of $1 \mathrm{~mm}$. The electrodes are two graphene films. Graphene has several advantages when used as electrodes including very low sheet resistance, high flexibility, excellent chemical stability, compatibility with the SIC film, strong interfacial charge transfer, high electron mobility and the ability to boost and drive high current density into the SIC film. The commercially available graphene electrodes were $25 \mu \mathrm{m}$ thick and had a sheet resistance of $2.8 \times 10^{-2} \Omega$ /square. The SIC sensing element consisted of a sodium-poly(sialate-siloxo) (Na-PSS) film. Na-PSS composites are essentially geopolymeric cements synthesized from alumino-silicate materials such as fly ash with a $\mathrm{Si} / \mathrm{Al}$ ratio of 2 [15]. Hence, in this work, the Na-PSS-based SIC sensing film with a Si/Al ratio of around 2 was synthesized from an alkaline solution and low calcium fly ash using a commonly used mix proportion [16]. The alkaline activator consisted of a mixture of sodium silicate $\left(\mathrm{Na}_{2} \mathrm{SiO}_{3}\right.$ with $29.4 \% \mathrm{SiO}_{2}, 14.7 \% \mathrm{Na} 2 \mathrm{O}$ and $\left.59.9 \% \mathrm{H}_{2} \mathrm{O}\right)$ and sodium hydroxide $(\mathrm{NaOH})$. The Na-PSS film was composed of $73 \%$ of fly ash and $27 \%$ alkaline activator. The alkaline activator to fly ash ratio was 0.37 and the ratio of $\mathrm{Na}_{2} \mathrm{SiO}_{3}$ to $\mathrm{NaOH}(12 \mathrm{M})$ was 2.4.

\subsection{Sensor fabrication}

Figure 2 shows the steps employed to manufacture the SIC sensor. The fly ash powder was first added to the alkaline solution and mixed for 1 minute with a DAC 150 high centrifugal mixer. Upon mixing, a layer of geopolymer of about $0.5 \mathrm{~mm}$ in thickness was deposited in a plastic mold. Subsequently, the two graphene electrodes of $15 \mathrm{~mm}$ x $20 \mathrm{~mm}$ equipped with conductive glue contact points were deposited and a second layer of geopolymer of about 0.5 mm was then added to achieve the total sensor's thickness of $1 \mathrm{~mm}$. With the contact points naked, the fabricated sensor was oven cured at $60^{\circ} \mathrm{C}$ for 24 hours and then left to cool down in room temperature prior to attaching the lead wires for characterization. In total 30 sensors were fabricated and evaluated in this study. Figure 3 shows an image of the fabricated SIC sensor. 


\subsection{Characterization and EIS measurements}

Energy-dispersive X-ray spectroscopy (EDX) and X-ray diffraction (XRD) analysis tools were performed to identify the mineralogical composition and chemical elements of the geopolymeric SIC film and the graphene electrodes. A scanning electron microscope (SEM) was employed to determine the morphology of the SIC film, graphene and graphene/SIC film interface.

The Electrical Impedance Spectroscopy (EIS) analysis method was employed to characterize the SIC sensor response to temperature. The sensor was placed in a controlled environmental chamber and EIS measurements were conducted at $-10,-5,5,20$ and $35{ }^{\circ} \mathrm{C}$ to determine its ionic conductivity, conduction mechanism, activation energy, dielectric constant and temperature sensitivity. In this test, the environmental chamber was heated from -10 to $35^{\circ} \mathrm{C}$ in 5 and $15{ }^{\circ} \mathrm{C}$ steps. For each step, the temperature was kept constant for 1 hour in order to achieve thermal equilibrium in the chamber. In total, ten sensors were evaluated. The EIS analysis method was also employed to evaluate the effect of load on the sensor's impedance and to determine the sensor's strain sensitivity at room temperature using the cantilever beam test setup shown in Fig. 4. The cantilever beam consisted of a fiberglass beam of $4 \mathrm{~mm}$ in thickness, $40 \mathrm{~mm}$ in width and $400 \mathrm{~mm}$ in length. The beam was clamped to a table at one end and the load was applied on the other end. The sensor was tightly bonded to the beam surface with a high strength adhesive agent over its gauge length (i.e., $60 \mathrm{~mm}$ ) to ensure full strain transfer to the sensor without any slippage. The sensor was connected to the EIS system. The beam was loaded from 10 to $40 \mathrm{~N}$ in 2, 3 and $5 \mathrm{~N}$ steps. Each load was held for 5 minutes to allow multiple measurements of the sensor's impedance. The effect of temperature on the strain sensor's response was not investigated during this experiment due to the small size of the environmental chamber. However, the effect of temperature on the strain sensor's response was negligible because the ambient room temperatures were constant 
over the duration of the bending tests. In total, 20 sensors were evaluated in these experiments.

The EIS measurements were performed over a frequency range of $0.04 \mathrm{~Hz}-5 \mathrm{kHz}$ using Gamry Interface 1000 Potentiostat. During measurement, a $10 \mathrm{mV}$ voltage was applied to the sensor and, the phase shift from the input signal and the amplitude of the AC current response were used to compute the real ( $Z$ ', resistive) and imaginary ( $Z^{\prime \prime}$, capacitive) components of the complex impedance $Z(\omega)=Z^{\prime}+j Z^{\prime \prime}$ as follows:

$$
\begin{aligned}
& R_{e}(Z)=Z^{\prime}=|Z(\omega)| \cos \alpha \\
& I_{m}(Z)=Z^{\prime \prime}=|Z(\omega)| \sin \alpha
\end{aligned}
$$

where $|Z(\omega)|$ is the impedance magnitude, $\omega$ is the operating frequency and $\alpha$ is the phase difference between the voltage and current (i.e. the phase shift).

\section{Experimental results}

\subsection{Morphology, mineralogical composition and chemical structure}

Figure 5 shows the SEM image, the EDX spectrum and the chemical composition of the geopolymeric SIC film. As can be seen, the matrix of the geopolymeric film consists of an alumino-silicate gel with unreacted fly ash particles. The SEM image shows that the geopolymeric film has a heterogeneous microstructure due to the variation in the physical, chemical, and mineralogical properties of the fly ash used in this study. The SEM image also shows a closed pore structure of the geopolymeric film. The pores are the result of the partially or fully reacted fly ash particles. The table in the EDX spectrum provides the average values of the detected chemical elements. From the obtained chemical elements, the $\mathrm{Si} / \mathrm{Al}$ ratio is 2.18 , implying that the geopolymeric film is a $3 \mathrm{D}$ framework in the form of sodium-poly(sialate-siloxo) (Na-PSS or $\mathrm{Na}$ (-Si-O-Al-O-Si-O-) with a molecule structure shown in Fig. 6 as proposed by Davidovits [17]. Other cations such as $\mathrm{K}^{+}$and $\mathrm{Ca}^{+}$are also present in the framework but in lesser proportions. The Na-PSS crystal contains electron vacancies or holes which control the electrical properties of the Na-PSS film. As a result, the 
main cation $\mathrm{Na}^{+}$hops onto these electron vacancies to balance the negative charge of $\mathrm{Al}$ as depicted in Fig 6.

The XRD pattern shown in Fig. 7-a shows that the geopolymer-based SIC film is a semicrystalline structure mainly composed of quartz (Q) at $2 \Theta$ of approximately $20,27,41,50,61$ and $78^{\circ}$, and mullite $(\mathrm{M})$ at $2 \Theta$ of approximately $15,31,36,44$ and $65^{\circ}$. These amorphous phases are typical of poly(sialate-siloxo) geopolymers. The XRD pattern of graphene (Fig. 7b) shows a sharp peak at $2 \Theta$ of $26.79^{\circ}$ with a $d$-spacing of $3.32 \AA$. These XRD characteristics are highly specific for the unique crystalline nature of graphene.

The morphology of the graphene electrode in Fig. 8 shows wrinkling and folding resulting from the processing of the graphene sheets. The morphology of the graphene/Na-PSS film interface is shown in Fig. 9. As shown, no delamination of the graphene electrodes is observed and the bond between the Na-PSS film and graphene appears to be strong. Although, graphene does not chemically bond with the alumino-silicate film, we hypostasize that the sialate-siloxo (-Si-O-Al-Si-O-) groups and water layers available in the Na-PSS film can form dipole-dipole bonds with graphene, thereby allowing the transfer of electrons between the two materials.

\subsection{Impedance and dielectric spectroscopy properties}

The Nyquist plot for the sensor shown in Fig. 10 indicates that the sensor exhibits a near pure-resistive behavior. Its impedance spectrum consists of two main parts: a flat line parallel to the real impedance axis in the high frequency region and a sloped line in the low frequency region. The flat line where the imaginary impedance is near zero can be attributed to both the intrinsic resistance of the Na-PSS film and the charge transfer resistance at the graphene electrode interface. The sloped line is related to Warburg ion diffusion process which is slowly controlled by the pore structure of the Na-PSS film and the flat graphene/NaPSS film interfaces. The intersection of the sloped line with the flat line at a cut-off frequency of about $100 \mathrm{~Hz}$ represents the true bulk resistance of the sensor which is about 
$330 \mathrm{k} \Omega$. The Bode plot of the impedance of the sensor depicted in Fig. 11 further confirms that the sensor behaves as a pure resistor because the total impedance is largely resistive over the frequency range of $100 \mathrm{~Hz}-5 \mathrm{kHz}$.

The Nyquist plots for the sensor at $-10,-5,5,20$ and $35^{\circ} \mathrm{C}$ are shown in Fig. 12. First, it can be observed that in the low frequency region, the shape of the three Nyquist spectra is similar to that at room temperature. However, in the high frequency region, the shape of the Nyquist spectrum at room temperature appears to change from a flat line to an incomplete semicircle. The incomplete semicircle can be attributed to a combined effect of the charge-transfer resistance at the flat graphene electrode interface and the double-layer capacitance. Second, it can also be observed that the impedance spectrum shrinks in size and shifts to the left, toward the imaginary axis (- $Z^{\prime \prime}$ axis) with increasing temperature. This means the real impedance $Z^{\prime}$ decreases with increasing temperature as a result of increased ion mobility and thermal activation of conducting species in the Na-PSS film.

Figures 13 and 14 depict the variation of the real and imaginary impedances with frequency at $-10,-5,5,20$ and $35^{\circ} \mathrm{C}$. As shown, both impedances decrease with increasing temperature and decrease with increasing frequency up to $100 \mathrm{~Hz}$. At higher frequencies, both impedances level off at all temperatures, but the imaginary impedance somewhat converges toward zero at all temperatures. This implies that the sensor behaves as a pure resistive temperature sensor in the frequency range of $100 \mathrm{~Hz}-5 \mathrm{kHz}$.

The frequency dependence of the dielectric constant is shown in Fig. 15. The dielectric constant $\epsilon^{\prime}$ was calculated using the following equation:

$$
\epsilon^{\prime}=\frac{d Z^{\prime \prime}}{2 \pi Z^{2} \epsilon_{0} A}
$$

Where $d$ is the distance between the electrodes, $\epsilon_{0}$ is permittivity of the free space and $A$ is the projected contact area of the graphene electrode. As can be seen, the dielectric constant increases with temperature and decreases with frequency. The decrease in the dielectric constant with frequency is due to the dielectric dispersion within the system as the dipoles are 
not able to rotate or oscillate sufficiently to follow the applied ac-field. The increase in the dielectric constant with temperature is attributed to the fact that increasing temperature enables the formation and orientation of dipoles which leads to higher dielectric constants.

\subsection{Ionic conductivity and activation energy}

The ionic ac-conductivity of the Na-PSS film was calculated using the following equations:

$$
\begin{gathered}
\sigma_{a c}=2 \pi f \epsilon^{\prime \prime} \epsilon_{0} \\
\epsilon^{\prime \prime}=\frac{d Z^{\prime}}{2 \pi Z^{2} \epsilon_{0} A}
\end{gathered}
$$

where $f$ is the frequency of the electric field and $\epsilon_{0}$ is the permittivity of the free space and $A$ is the projected contact area of the graphene electrode. The room temperature ionic conductivities of the sensor are $1.54,1.64$ and $1.72\left(10^{-2} \mathrm{~S} / \mathrm{m}\right)$ at $0.1,1$ and $5 \mathrm{kHz}$, respectively. Figure 16 shows the variation of $\log$ conductivity $\left(\sigma_{a c}\right)$ with inverse of temperature $(1 / T)$. As shown, the sensor exhibits a strong temperature-conductivity behavior governed by the Arrhenius equation:

$$
\sigma_{a c}=\sigma_{0} \exp \left(\frac{-E_{a}}{E_{B} T}\right)
$$

where $\sigma_{0}, E_{a}$ and $E_{B}$ are the pre-exponential factor, the activation energy and the Boltzmann constant, respectively. The activation energy $E_{a}$ is defined as the slope of the Arrhenius plot times the Boltzmann constant $E_{B}$. As depicted in Fig. 16, the slope $S$ of each data set is determined using the linear least squares fitting method and the corresponding activation energy is then calculated from the fit data. It is interesting to note that the frequency has slight effect on the activation energy. The calculated average activation energy value for the sensor is displayed in Fig. 16. The average value obtained for the sensor is $0.156 \mathrm{eV}$ in the frequency range of $100 \mathrm{~Hz}-5 \mathrm{kHz}$. 


\subsection{Sensitivity to temperature}

The sensor's response to temperature at three representative frequencies of $0.1,1$ and $5 \mathrm{kHz}$ is shown in Fig. 17. At these frequencies, the sensor shows very low imaginary impedance values. As a result, the fractional change in the real impedance $\Delta Z^{\prime} / Z_{0}^{\prime}$ is used as the overall response of the sensor. Figure 17 indicates that $\Delta Z^{\prime} / Z_{0}^{\prime}$ is a linear function of temperature being $\Delta Z^{\prime} /_{Z_{0}^{\prime}}=\alpha_{T e m p} T$, where $\alpha_{T e m p}$ is the temperature sensitivity coefficient and $Z_{0}^{\prime}$ is the initial real impedance measured at $-10^{\circ} \mathrm{C}$. Fig. 17 also indicates that the temperature sensitivity of the sensor $\alpha_{\text {Temp }}$ is independent of frequency with a value as high as $0.0198 /{ }^{\circ} \mathrm{C}$.

\subsection{Sensitivity to tensile strain}

The effect of the applied load on the Nyquist plot of the sensor's impedance is displayed in Fig. 18. From this figure, it can be clearly seen that the applied load does not significantly alter the shape of the impedance spectra, but shifts them progressively to the right along the real impedance. This means the impedance of the sensor at the cusp frequency of $100 \mathrm{~Hz}$ (i.e., true bulk resistance) at $0 \mathrm{~N}$ increases as the load increases. To better understand this piezoresistive response, the effect of the applied load on both imaginary and real impedances is plotted in Figs. 19 and 20, respectively. It can be observed from Fig. 19, that the imaginary impedance does not change, while the real impedance increases with increasing load (Fig. 20). This implies that the impedance response of the sensor is largely dominated by the real impedance of the Na-PSS film. The increase in the real impedance with increasing load is attributed to the effect of load-induced strain and deformation on the ion diffusion in the NaPSS film. Figure 21 plots the fractional change in the real impedance $\Delta Z^{\prime} / Z_{0}^{\prime}$ against the applied load for two representative frequencies of 1 and $5 \mathrm{kHz}$. As shown, the sensor exhibits a strong linear trend of increase of the real impedance with increasing load. The frequency has little effect on the sensor's response . This again confirms the resistive-like behavior of the sensor. The effect of load induced tensile strain on the electrical properties of 
SICs is still not well understood, however, it is believed that the strain-induced microstructural changes alter the electronic band structure of SICs, thereby modifying both the mobility of the charge carriers and the electrical conductivity [14]. Here, we hypothesize that the tensile strain increases the length of ion diffusion paths in the Na-PSS lattice structure (i.e., distances between the electron holes) allowing for slower migration of $\mathrm{Na}^{+}$ cation from one site to another. This phenomenon decreases the ionic conductivity of the sensor. As the tensile strain increases, the tortuosity of ion diffusion becomes predominant. This leads to a more obstructed ion transport mechanism and the ionic conductivity further decreases as a result. The decrease in the ionic conductivity of the sensor could also be the result of the change in the pore morphology within the Na-PSS film. The tensile strain changes the pore constriction, thereby impeding the ion movement and hence the ionic conductivity of the sensor decreases as a consequence.

The strain sensitivity of piezoresistive sensors is often defined as the gauge factor (GF), which relates the fractional change in resistance $\Delta R / R_{0}$ to the applied strain $\varepsilon$ where, $\Delta R$ is the change in the electrical resistance and $R_{0}$ is the initial resistance. Because the sensor is somewhat behaving as a resistor in the frequency range of $100 \mathrm{~Hz}-5 \mathrm{kHz}$, i.e., $\Delta Z^{\prime} / Z_{0}^{\prime} \approx$ $\Delta R / R_{0}$, the GF equation can be written as:

$$
G F=\frac{\frac{\Delta \mathrm{R}}{R_{0}}}{\varepsilon} \approx \frac{\frac{\Delta Z^{\prime}}{Z_{0}^{\prime}}}{\varepsilon}
$$

The load-induced strain $\varepsilon$ in Eq. (7) can be estimated as the strain at the top surface of the beam assuming a perfect bond between the beam and the sensor. The strain $\varepsilon$ at the top of the beam can be derived from the cantilever beam theory as [2]:

$$
\varepsilon=\frac{(L-a) P c}{I E}
$$


Where $I$ is the $2^{\text {nd }}$ moment of area of the beam, $E$ is the modulus of elasticity of the beam, $L$ is the length of the beam, $a$ is the distance from the center of the strain sensor to the fixed end of the cantilever beam, $P$ is the applied load and $c$ is the distance from the centroid of the cantilever beam's cross-sectional area to the top surface of the beam where the SIC strain sensor is bonded. In Fig. 22, $\Delta Z^{\prime} / Z_{0}^{\prime}$ is plotted against the computed tensile strain $\varepsilon$ in the senor. The GF values obtained from the linear fits are displayed in the figure as 358 and 344 at 1 and $5 \mathrm{kHz}$, respectively.

\section{Discussion}

In this paper, we show that a highly sensitive and low-cost Na-PSS-based SIC sensor can be synthesized from graphene and waste materials such as fly ash for SHM applications. The proposed SIC sensor exhibits fast response to temperature and tensile strain. The sensor relies on the hopping of alkali ions to conduct current. The sensor exhibits high value dielectric constants where for example at $0.04 \mathrm{~Hz}$ and $20^{\circ} \mathrm{C}$, the dielectric constant is $1.5 \mathrm{x}$ $10^{8}$, suggesting that the Na-PSS film is a high-k dielectric material. The Na-PSS is a good ionic conductor; its room temperature ionic conductivities are in the range of recently reported sodium and lithium-based SICs, which achieved conductivities between $10^{-3}$ and 12 $\mathrm{mS} / \mathrm{cm}$ at room temperature [10-12]. The sensor also exhibits high temperature sensitivity coefficient. The highest measured temperature sensitivity is about $21.5 \mathrm{k} \Omega /{ }^{\circ} \mathrm{C}\left(\alpha_{\text {Temp }}=\right.$ $0.0198 /{ }^{\circ} \mathrm{C}$ and $Z_{0}^{\prime}=1.085 \mathrm{M} \Omega$ at $-10{ }^{\circ} \mathrm{C}$ ). This indicates that even small temperature changes cause a significant change in the sensor's impedance which makes the sensor an ideal thermistor for accurate temperature measurement. Although the strain limit was not determined, the sensor can measure strains up to $1300 \mu \varepsilon$ which is comparable or higher than commercial strain sensors. The measured GF values for the sensor are slightly affected by frequency and are much higher than those of commercial metallic foil (typically 2 to 5) and semiconductor (typically around 100) strain gauges. High GF values means that small strains produce large changes to the sensor's response. This is particularly attractive as 
sensitivity to small tensile strains enables the sensor to detect micro cracks in structures which usually occur in brittle materials at low tensile strain levels. Although the effects of temperature and load on the sensor's response were evaluated separately, its strain sensitivity will likely be affected by temperature and vice-versa. To obtain temperature-compensated tensile strain measurements, the temperature effect can be compensated by measuring temperatures using a control sensor isolated from mechanical strain. The sensor's response to temperature and strain will also be affected by other surrounding environments such as changes in local humidity and chemical contamination. These effects could be minimized by hermetically packaging the sensor, but the tradeoff is a slight reduction in the sensor's sensitivity. Multiple sensors can be located in areas of a structure where crack growth, high deformation and temperature are known to occur. The proposed sensor can easily be manufactured with long gauge lengths to monitor macro regions of a structure.

Although cracks in brittle materials are generally attributed to tensile strains, further work is needed to characterize the sensor's response under compressive loading. Further work is also needed to determine the repeatability, stability, accuracy and hysteresis behavior of the sensor under cyclic loading and, characterize the sensor's response when embedded or retrofitted onto structural elements.

\section{Conclusions}

In this work, a low-cost and sustainable graphene/SIC sensor for measuring temperature and strain in structures has been designed and characterized for the first time. The advantages of the proposed sensor over current piezoresistive sensors include high sensitivity, low-cost and a simple manufacturing process. The temperature characterization revealed that the sensor was able to measure temperature with high sensitivity (as high as $21.5 \mathrm{k} \Omega /{ }^{\circ} \mathrm{C}$ ) in the temperature range of -10 to $35^{\circ} \mathrm{C}$. As such, the sensor can be used for high precision temperature sensing in structures. The piezoresistive characterization revealed that the sensor was able to measure tensile strains up to $1300 \mu \varepsilon$ with extremely high GF (as high as 
358), thus making it suitable for measuring very small tensile strains. By careful package design and electrode placement, the proposed sensor can easily be manufactured with long gauge lengths to measure temperature and bulk strains in structures. The developed sensor is relatively inexpensive which can be embedded or retrofitted onto existing structures such as bridges, pipelines, buildings and wind turbines to monitor their long term health for improved integrity and sustainability. This not only reduces the costs associated with their maintenance and repair but also can extend their service life.

\section{References}

1. F. Ubertini, S. Laflamme, H. Ceylan, A. L. Materazzi1, G. Cerni1, H. Saleem, A. D’Alessandro and A. Corradini 2014 Novel nanocomposite technologies for dynamic monitoring of structures: a comparison between cement-based embeddable and soft elastomeric surface sensors, Smart Materials and Structures 23, DOI: 10.1088/09641726/23/4/045023.

2. Kang I, Schulz MJ, Kim JH, Shanov V and Shi D 2006 A carbon nanotube strain sensor for structural health monitoring Smart Materials and Structures 15 737-748, DOI: 10.1088/0964-1726/15/3/009.

3. Dharp P, Li Z, Nagarajaiah S and Barrera EV 2004 Nanotube film based on singlewall carbon nanotubes for strain sensing Nanotechnology 15, 379-82, DOI: 10.1088/0957-4484/15/3/026.

4. Jing Z, Yu G, and Xia SD 2013 Review of graphene-based strain sensors Chinese Phys. B 5, DOI: 10.1088/1674-1056/22/5/057701.

5. Deepak A, Srinivasan N, Karthik V, Ramya S, Ganesan SV, Shankar P 2015 Graphene based polymer strain sensors for non-destructive testing Advanced Materials. Research 1101, 314-317. 
6. Fu X, Lu W and Chung DDL 1998 Improving the strain-sensing ability of carbon fiber-reinforced cement by ozone treatment of the fibers Cement and concrete Research 2, 183-187, DOI: 10.1016/S0008-8846(97)00265-2.

7. Yu X and Kwon E 2009 Carbon-nanotube/cement composite with piezoresistive property Smart Materials and Structures 18, DOI: 10.1088/0964-1726/18/5/055010.

8. Azhari F and Banthia N 2012 Cement-based sensors with carbon fibers and carbon nanotubes for piezoresistive sensing Cement and Concrete Composites 7, 866-873, DOI: 10.1016/j.cemconcomp.

9. Howser RN, Dhonde HB and Mo YL 2011 Self-sensing of carbon nanofiber concrete columns subjected to reversed cyclic loading, Smart Materials and Structures 20, DOI: 10.1088/0964-1726/20/8/085031.

10. Inaguma Y, Chen L 1, Itoh M and Nakamura T 1994 Candidate compounds with perovskite structure for high lithium ionic conductivity Solid State Ionics, 196-202, DOI: 10.1016/0167-2738(94)90309-3.

11. W. D. Richards WD, Tsujimura T, Miara LJ, Wang Y, Chul Kim J, Ong SP, Uechi I, Suzuki N and Ceder G 2016 Design and synthesis of the superionic conductor Na10SnP2S12, Nature Communications DOI: 10.1038/ncomms11009.

12. Wang Y, Richards WD, Ong SP, Miara LJ, Kim JC, Mo1 Y, Ceder G 2015 Design principles for solid-state lithium superionic conductors Nature Materials DOI: 10.1038/NMAT4369.

13. Xiao R, Li RH and Chen L 2015 High-throughput design and optimization of fast lithium ion conductors by the combination of bond-valence method and density functional theory Scientific Reports DOI: 10.1038/srep14227.

14. Rowe ACH, Donoso-Barrera A, Renner CH and Arscott S 2008 Giant room temperature piezoresistance in a metal-silicon hybrid structure, Physical Review Letters DOI:10.1103/PhysRevLett.100.145501. 
15. Yunsheng Z, We Sun, Zuquan J, Hongfa Y, Yantao J 2007 In situ observing the hydration process of K-PSS geopolymeric cement with environment scanning electron microscopy Materials Letters 61 1552-1557.

16. Talha J , Obada K, Amar K, Jarvis B 2015 A mix design procedure for low calcium alkali activated fly ash-based concretes, Construction and Building Materials 79,301310.

17. Davidovits J, Geopolymer Chemistry and Applications, Institute Geopolymer, SaintQuentin, France. $4^{\text {th }}$ Ed., (2015). 

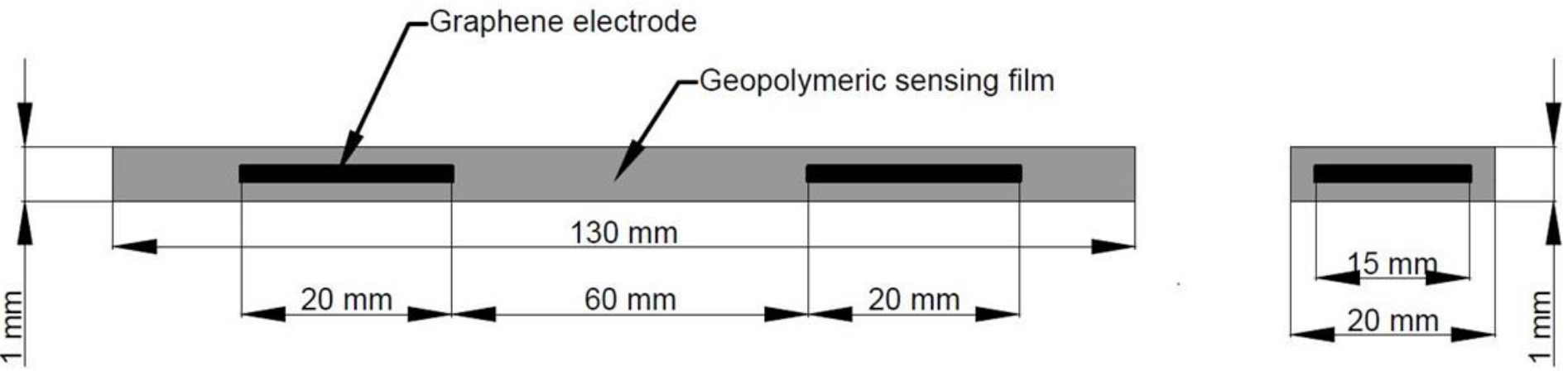

Figure 1: Layout of the sensor

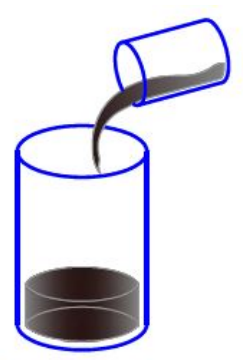

1. Fly Ash + Alkaline

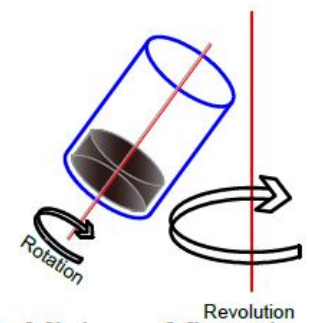

2. Mixing of fly ash with alkaline solution

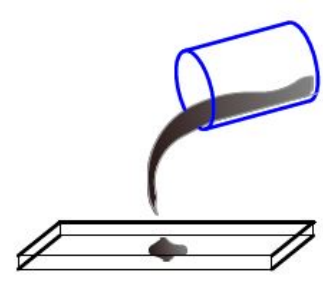

3. Deposition of 1 st layer of geopolymer

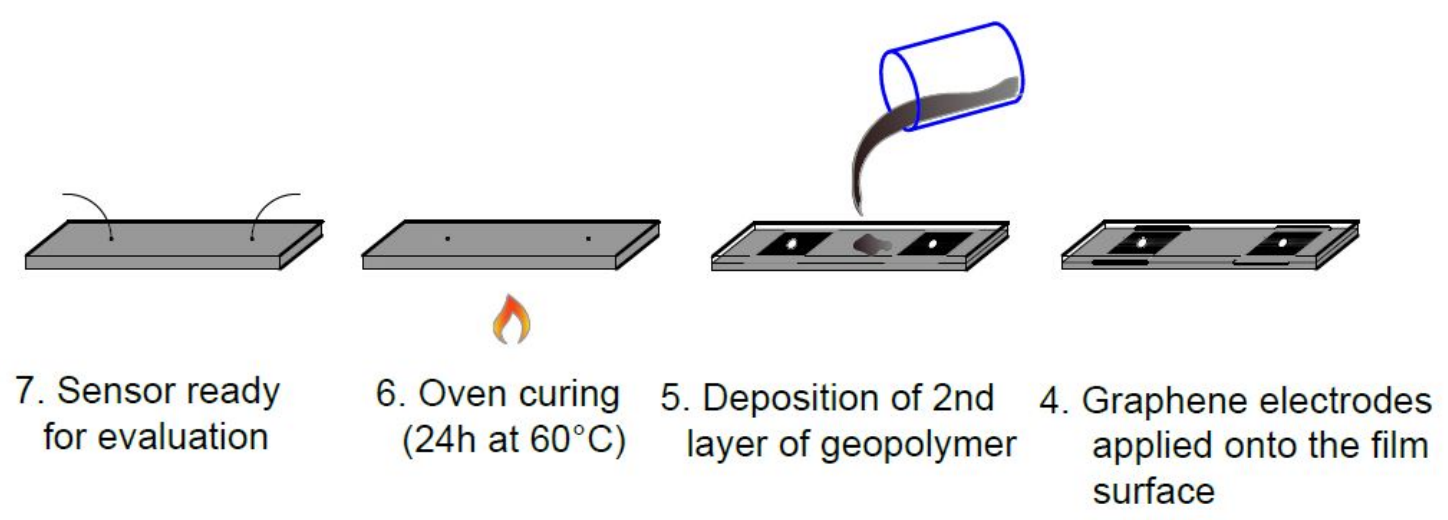

Figure 2: Schematic of the process used for the manufacture of the sensor 


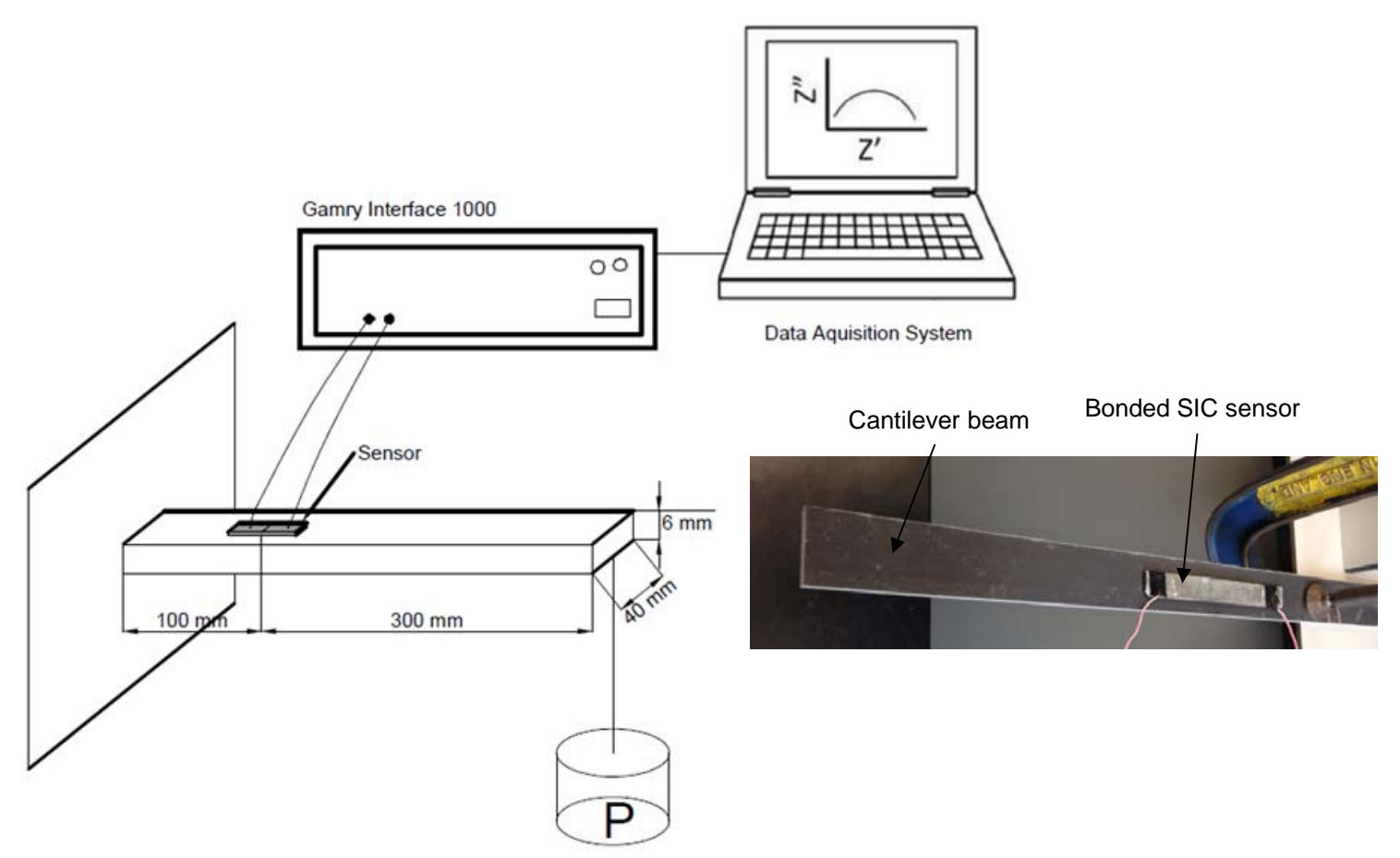

Figure 4: Test setup used to measure the sensor's response to tensile strain

\section{Na-PSS sensing film}

\section{Conductive glue leads}

Figure 3: Image of a fabricated sensor for temperature and strain measuring 
a)
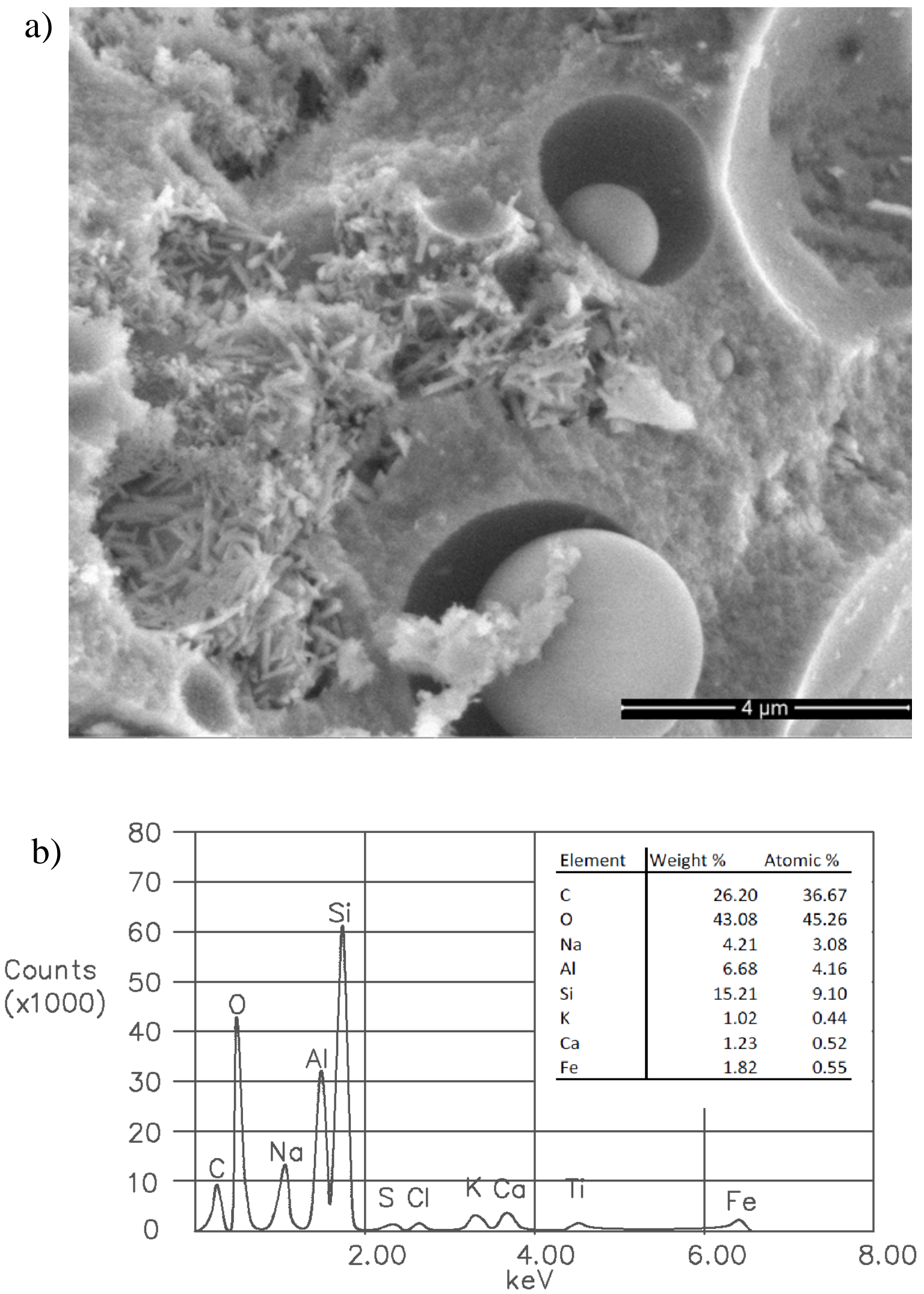

Figure 5: a) SEM image of the geopolymeric Na-PSS film, b) corresponding EDX spectrum describing the elemental distribution 


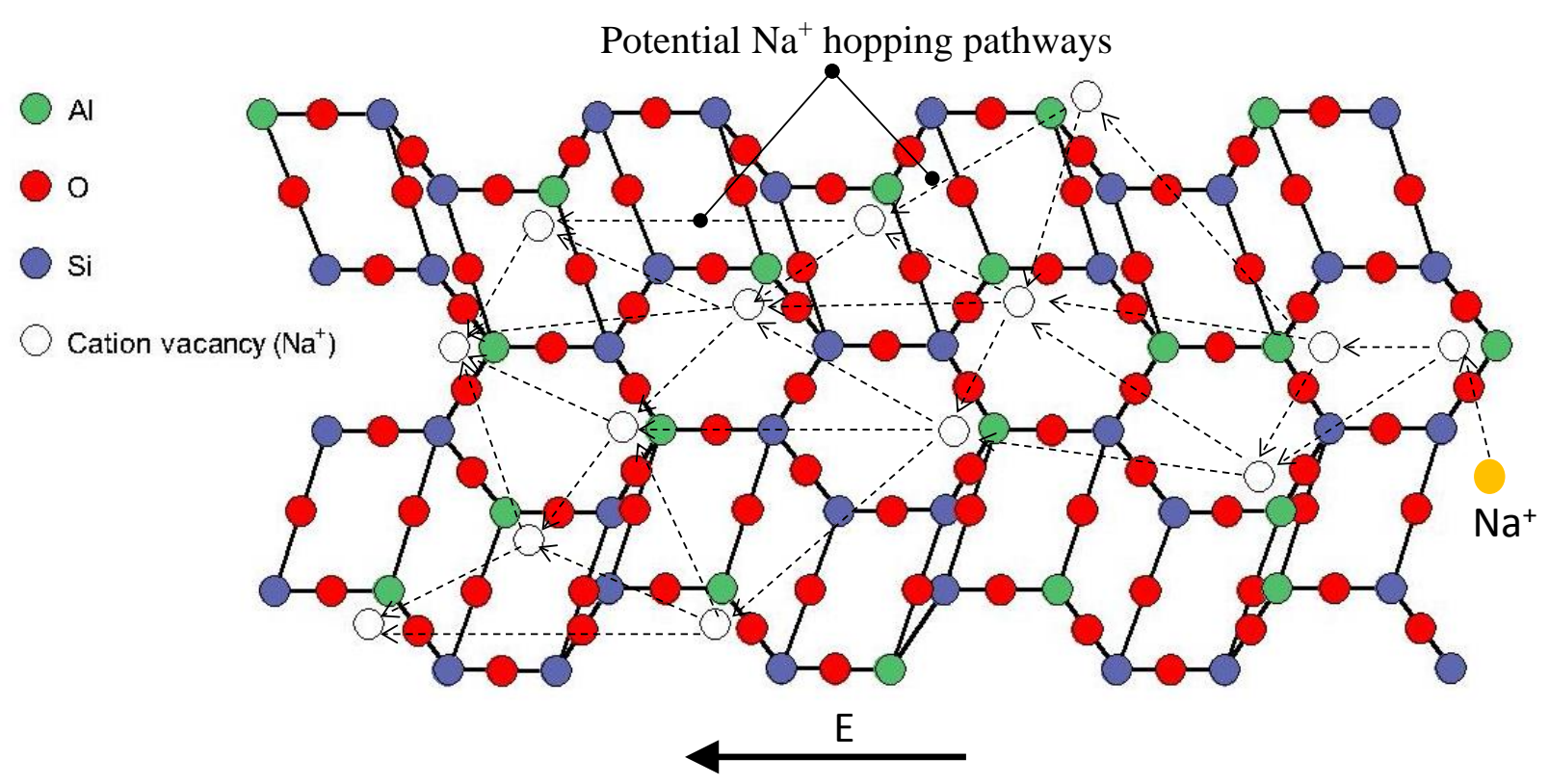

Figure 6: Typical 3D crystal structure of the Na-PSS film and hopping of $\mathrm{Na}^{+}$under electric field 

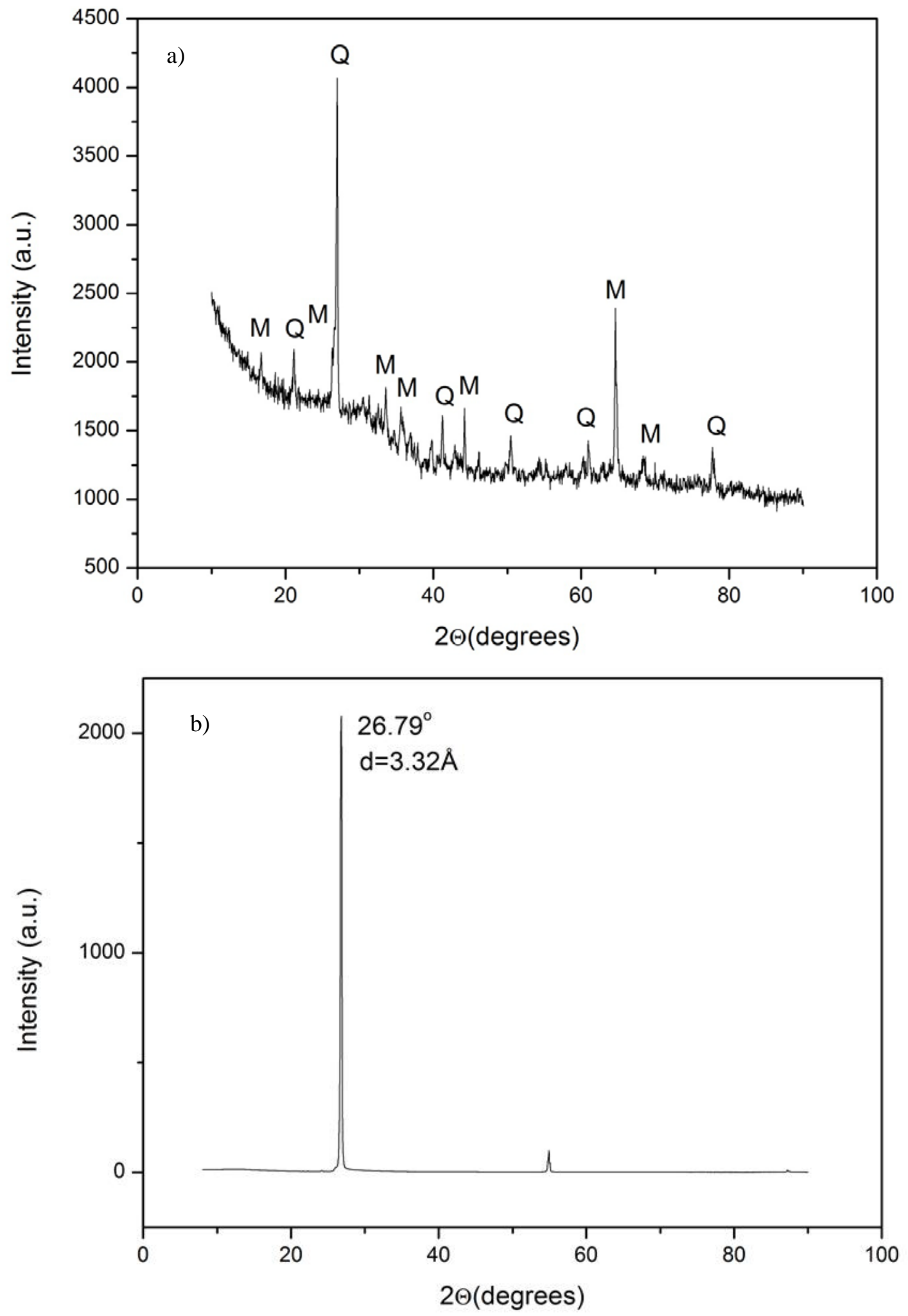

Figure 7: XRD patterns. a) geopolymeric Na-PSS film and b) graphene electrodes 


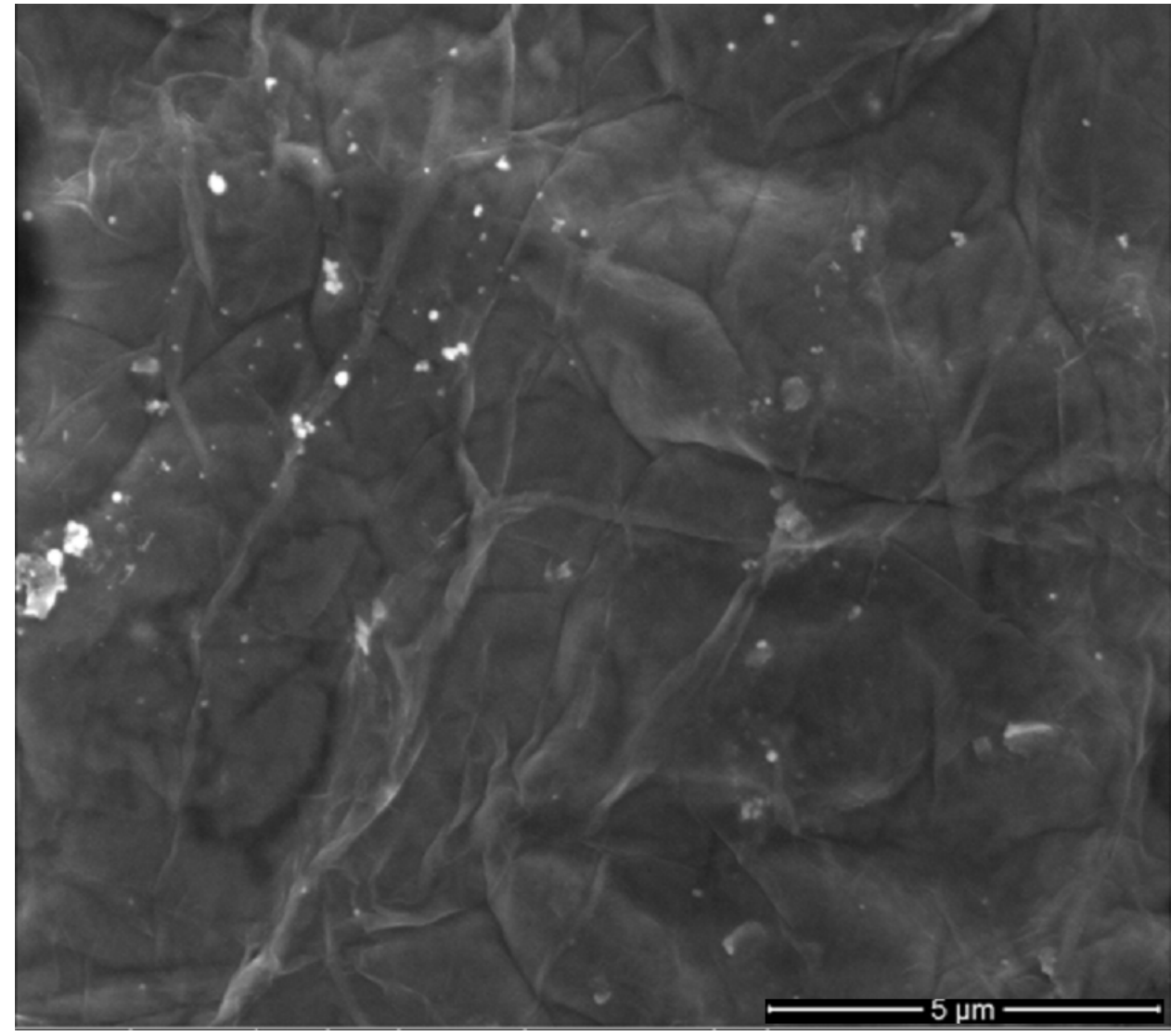

Figure 8: SEM image showing wrinkles and folds in the graphene electrodes 

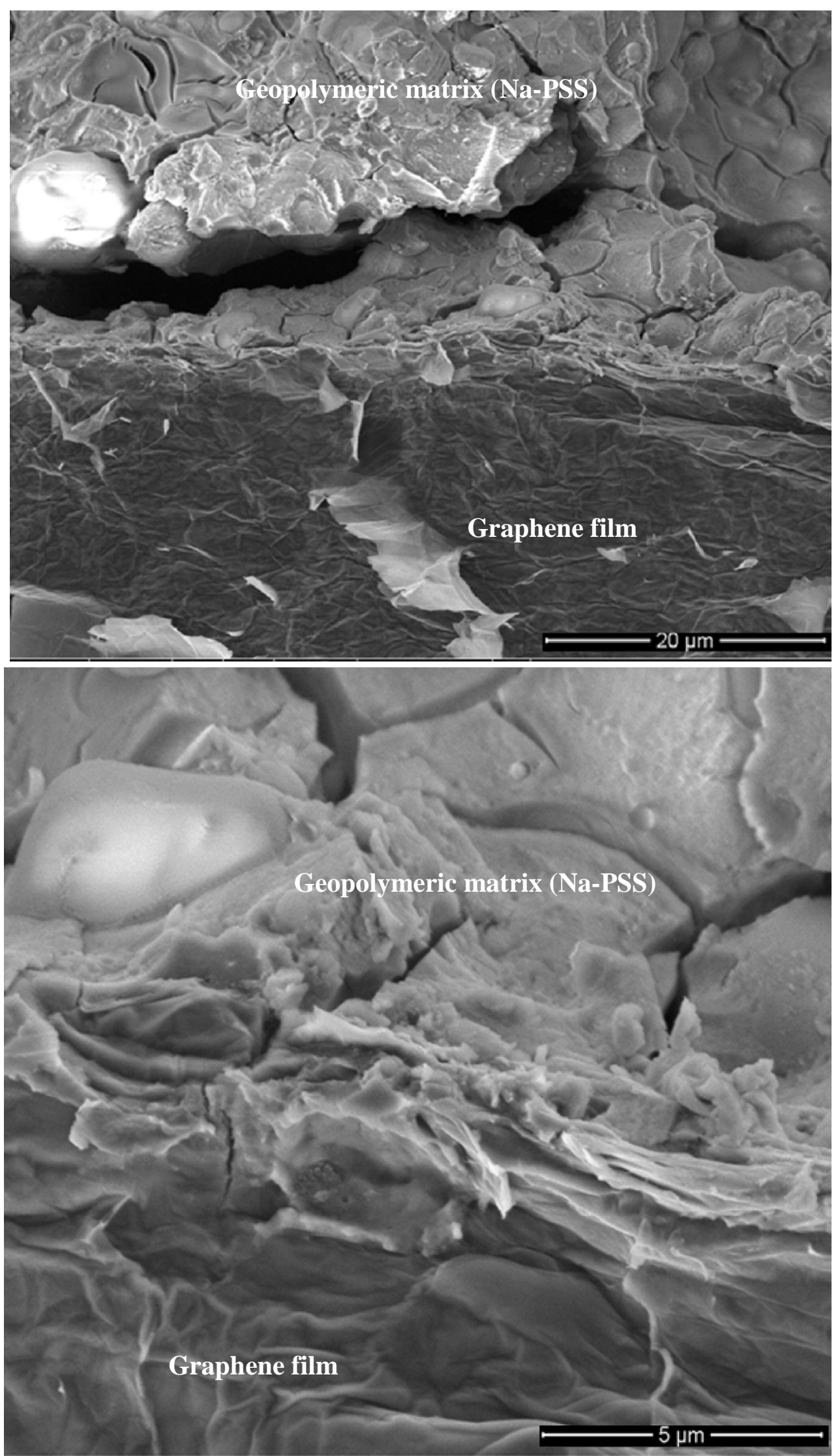

Figure 9: SEM image of the graphene/Na-PSS film interface, a) $20 \mu \mathrm{m}$ resolution and b) 5 $\mu \mathrm{m}$ resolution 


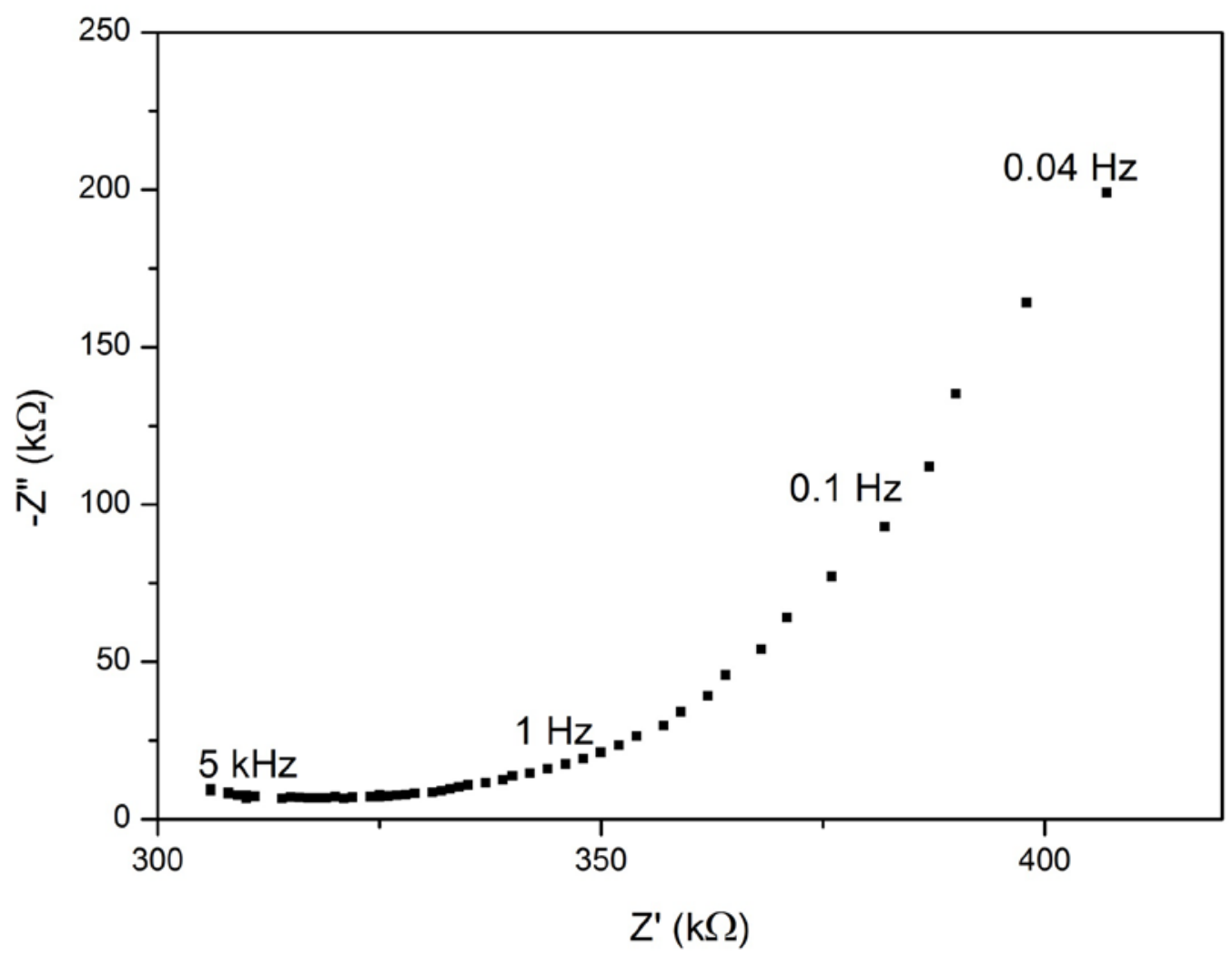

Figure 10: Nyquist plot for the SIC sensor at room temperature

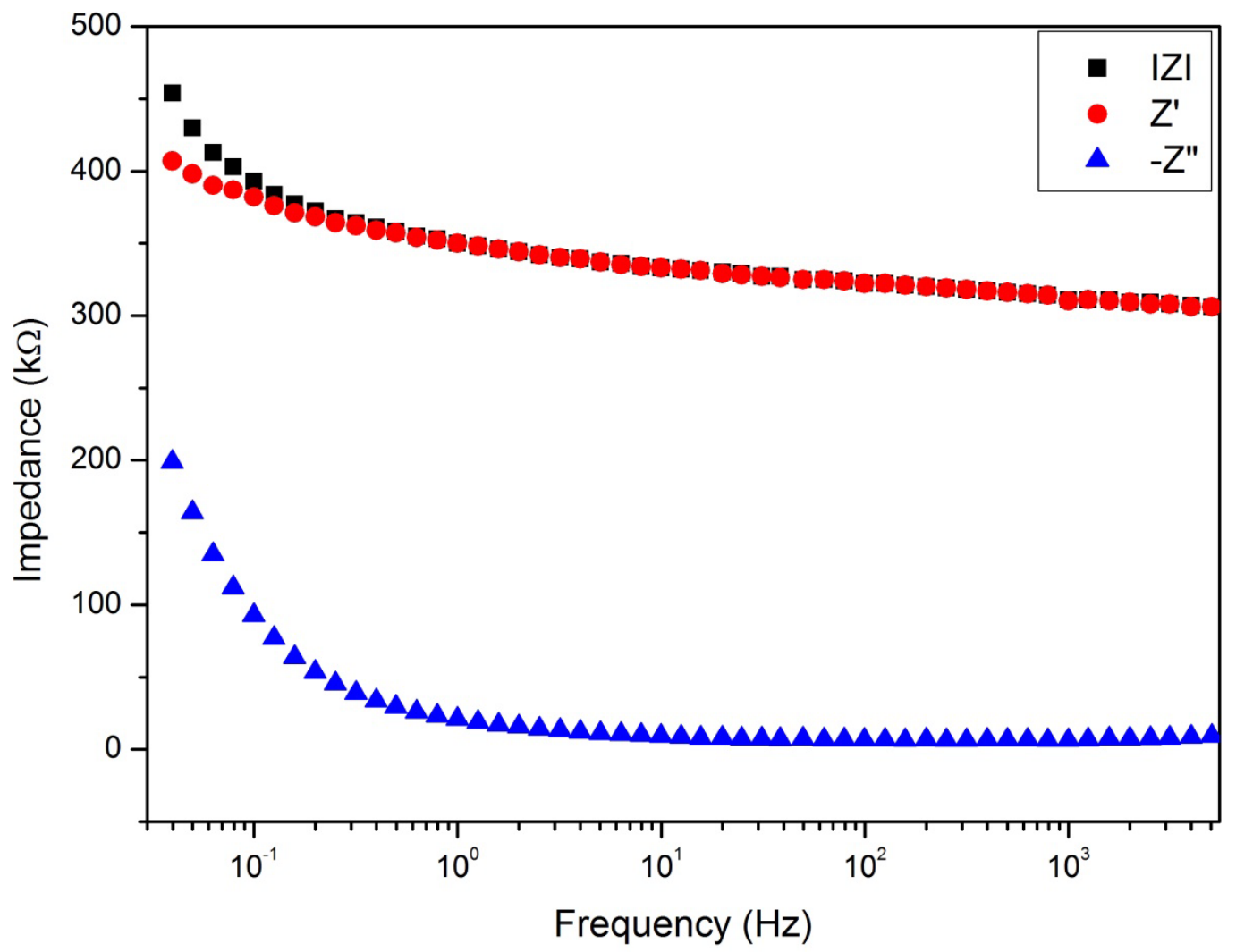

Figure 11: Bode plot of the impedance for the SIC sensor at room temperature 


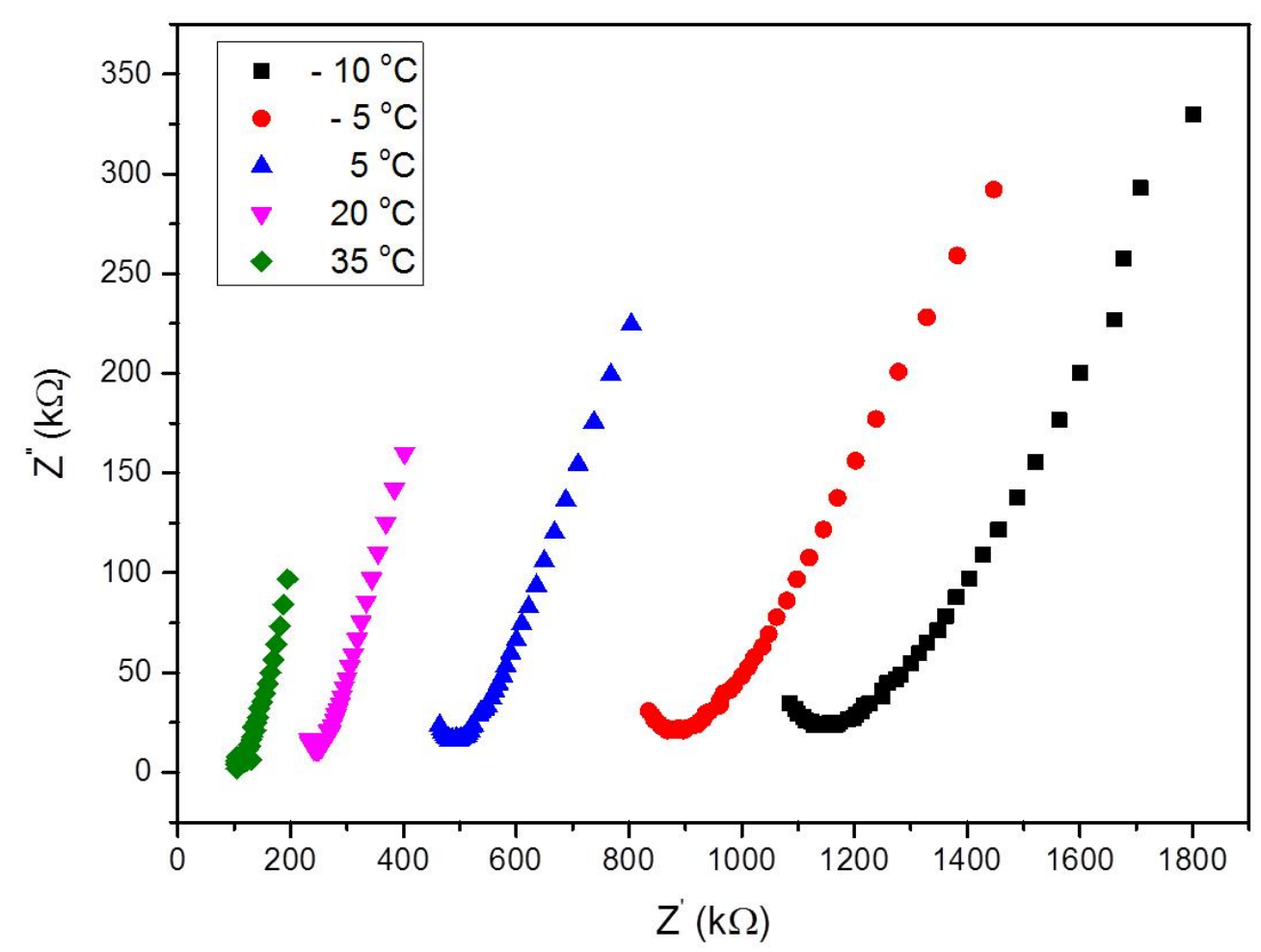

Figure 12: Nyquist plots for the SIC sensor at different temperatures

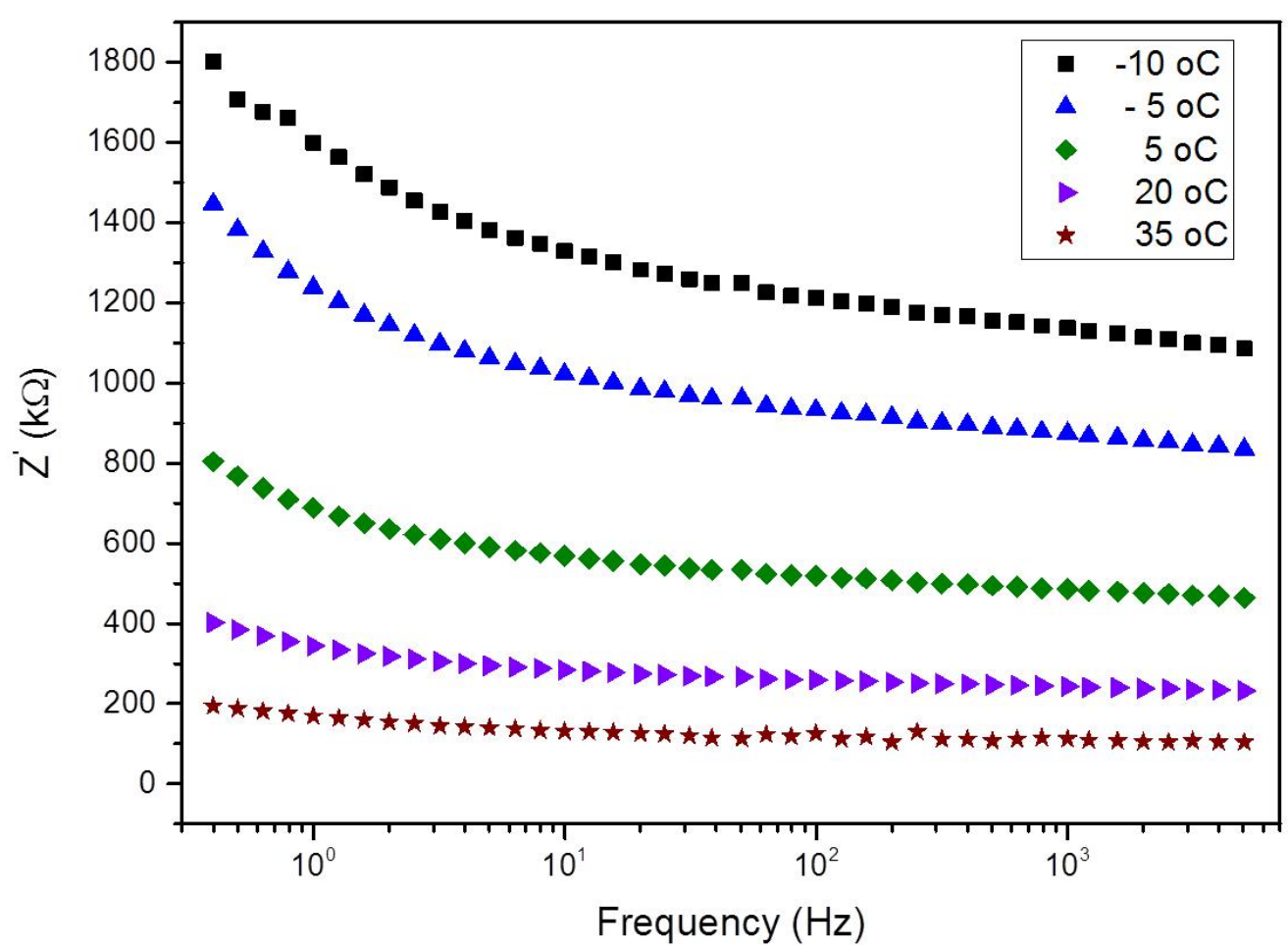

Figure 13: Bode plots of the real impedance for the SIC sensor at different temperatures 


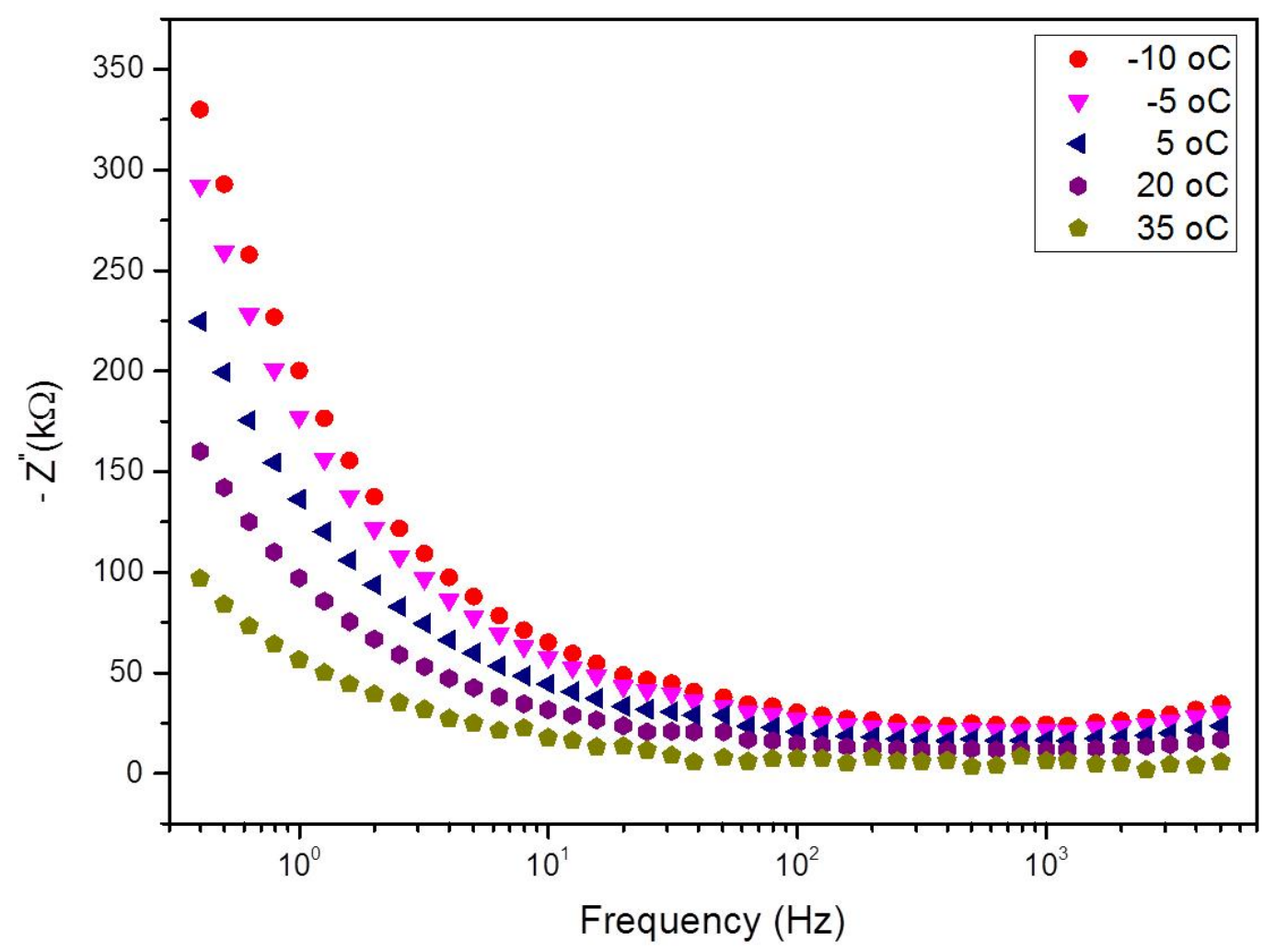

Figure 14: Bode plots of the imaginary impedance for the SIC at different temperatures

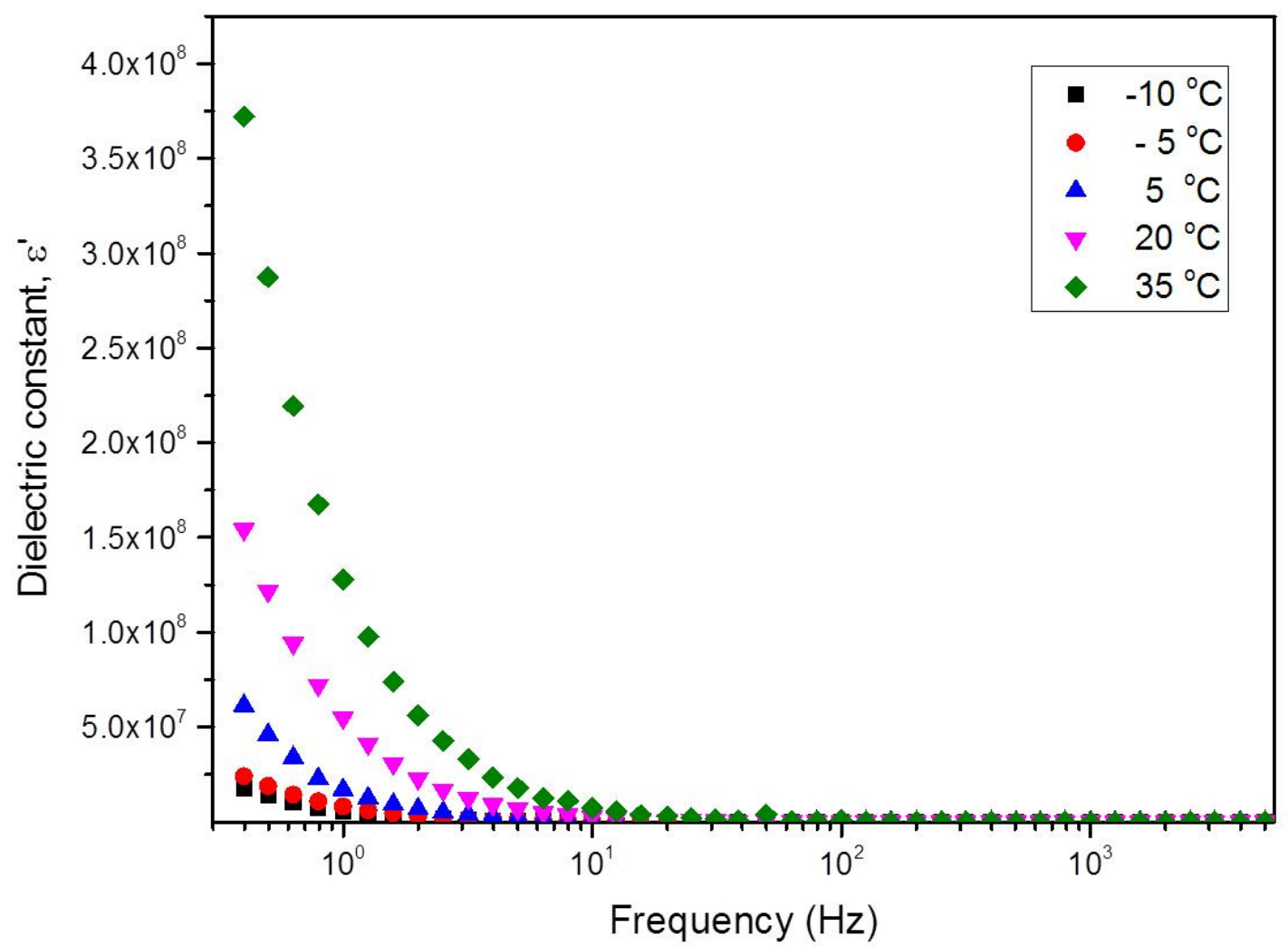

Figure 15: Dielectric constants of the sensor at different temperatures 


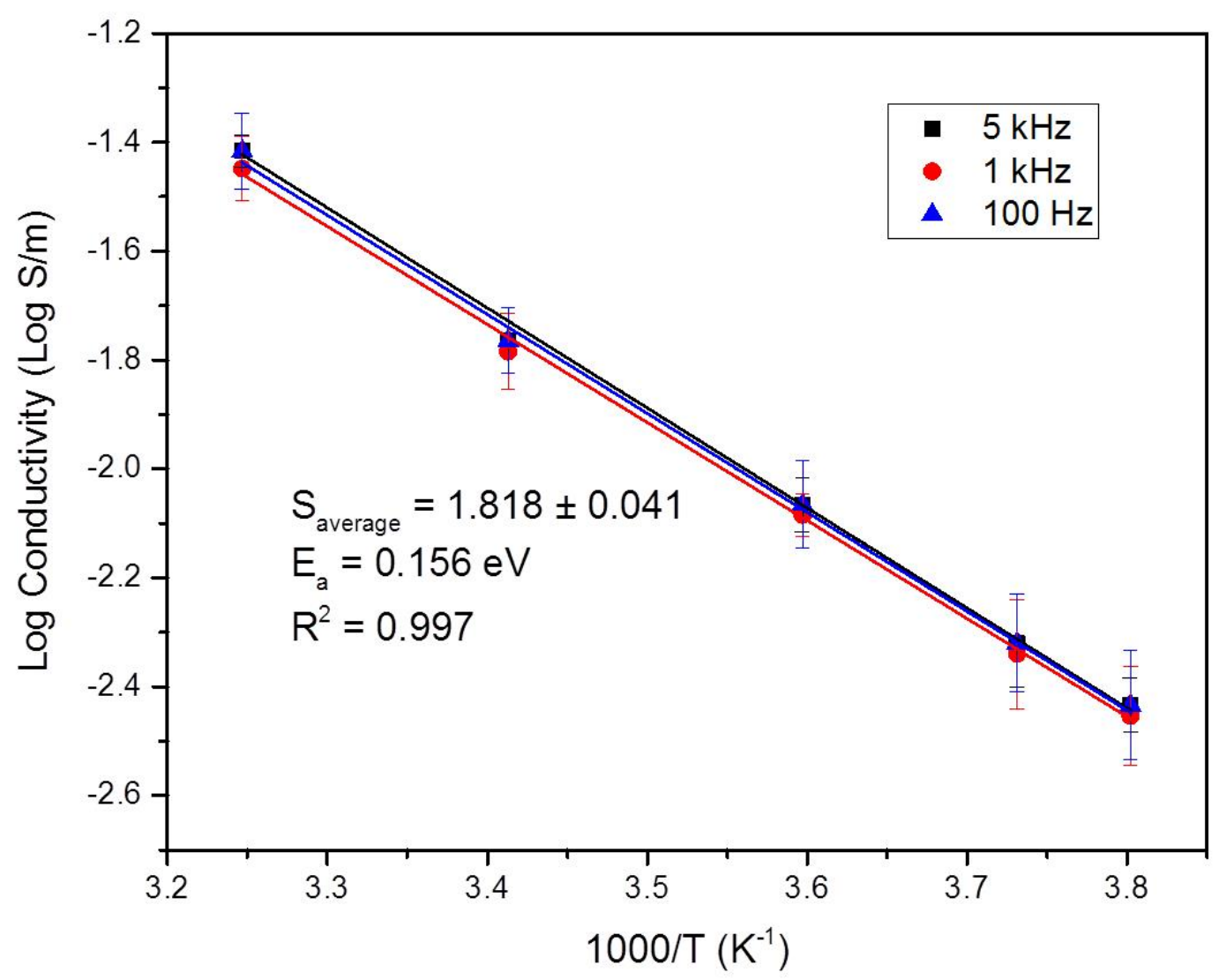

Figure 16: Arrhenius plot of the ac-conductivity for the SIC sensor at different frequencies

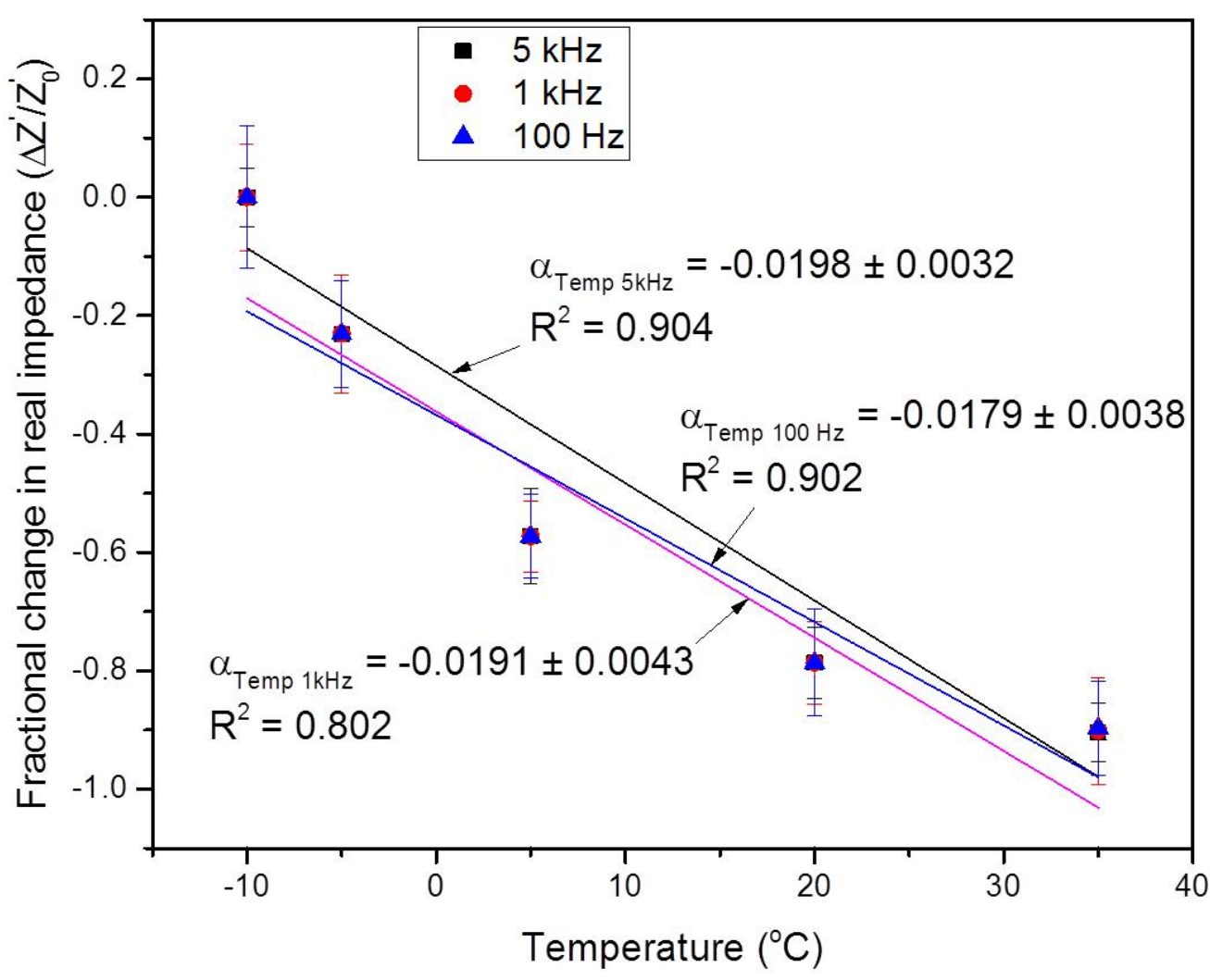

Figure 17: Temperature dependence of the fractional change in the real impedance relative to $5^{\circ} \mathrm{C}$ at different frequencies. 


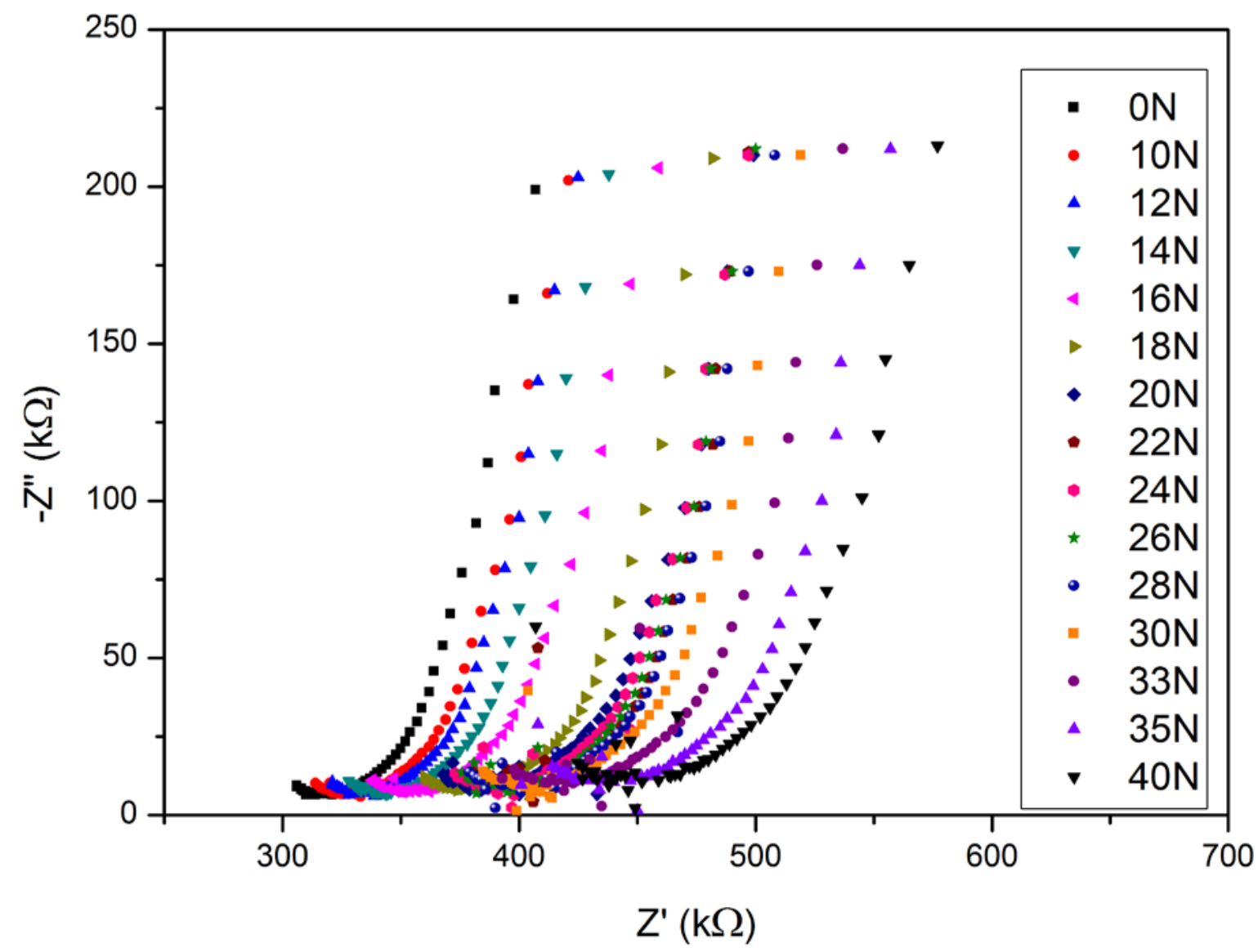

Figure 18: Nyquist plots of the impedance for the SIC sensor at different loads

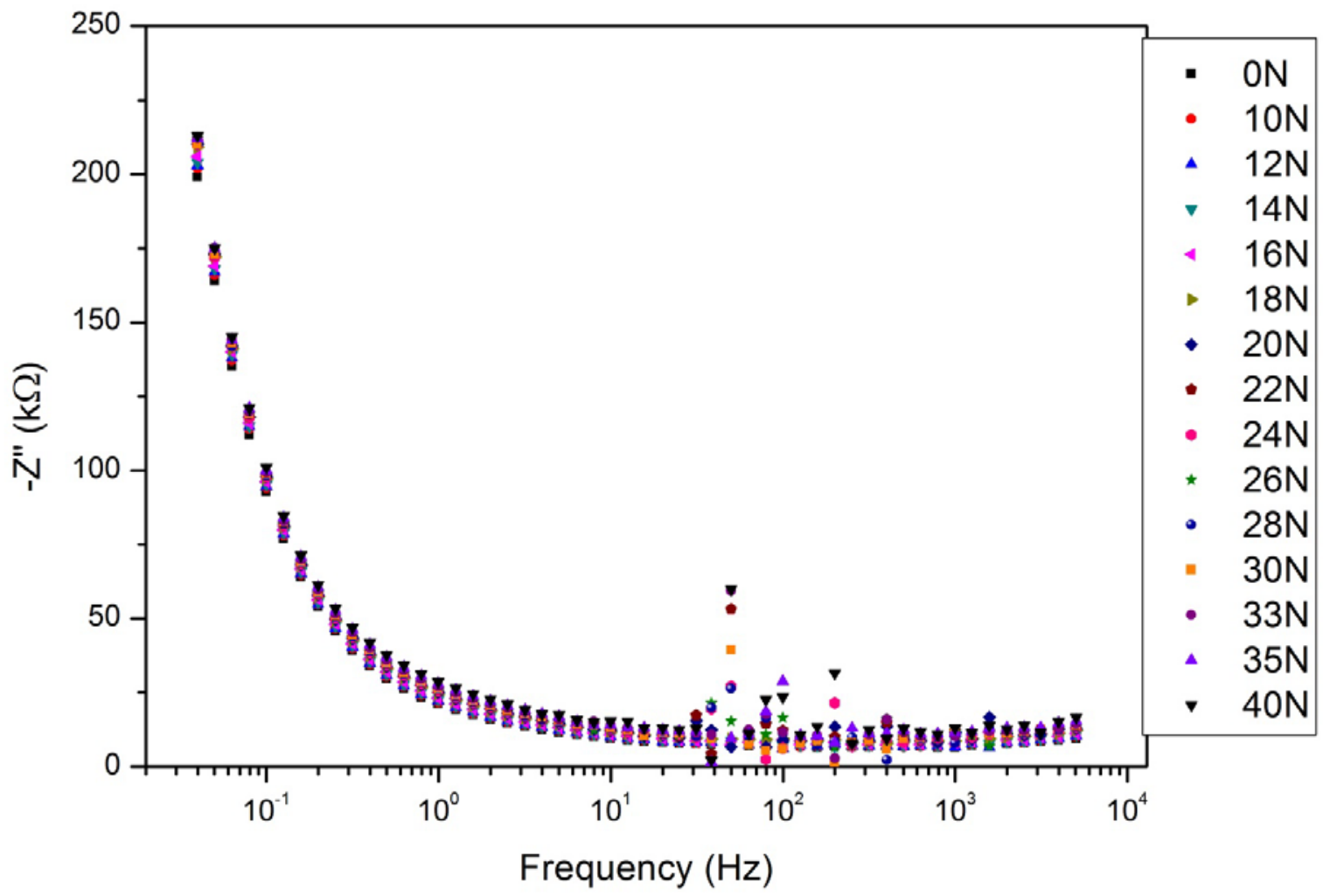

Figure 19: Bode plots of the imaginary impedance for the SIC sensor at different loads 


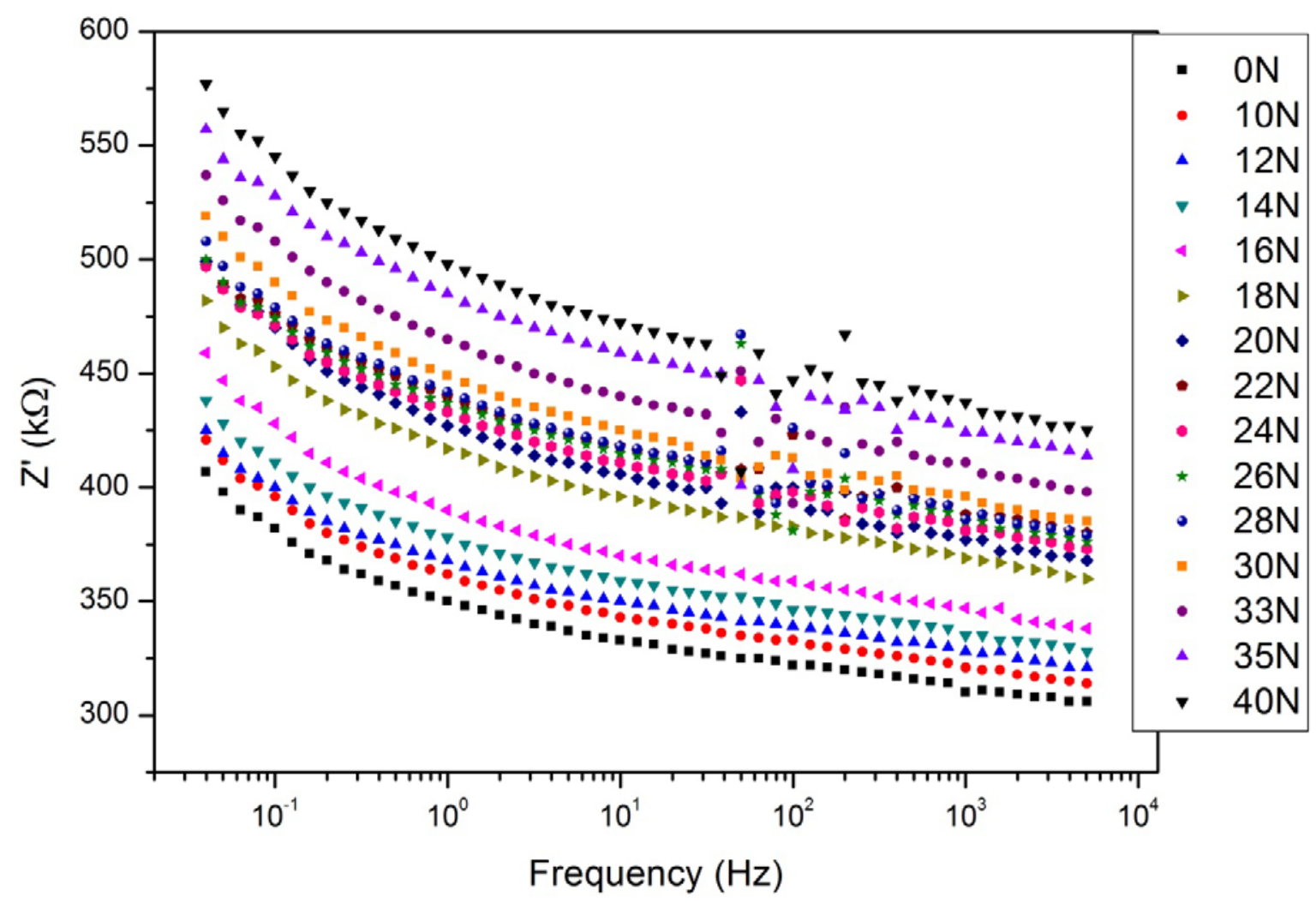

Figure 20: Bode plots of the real impedance for the sensor at different loads

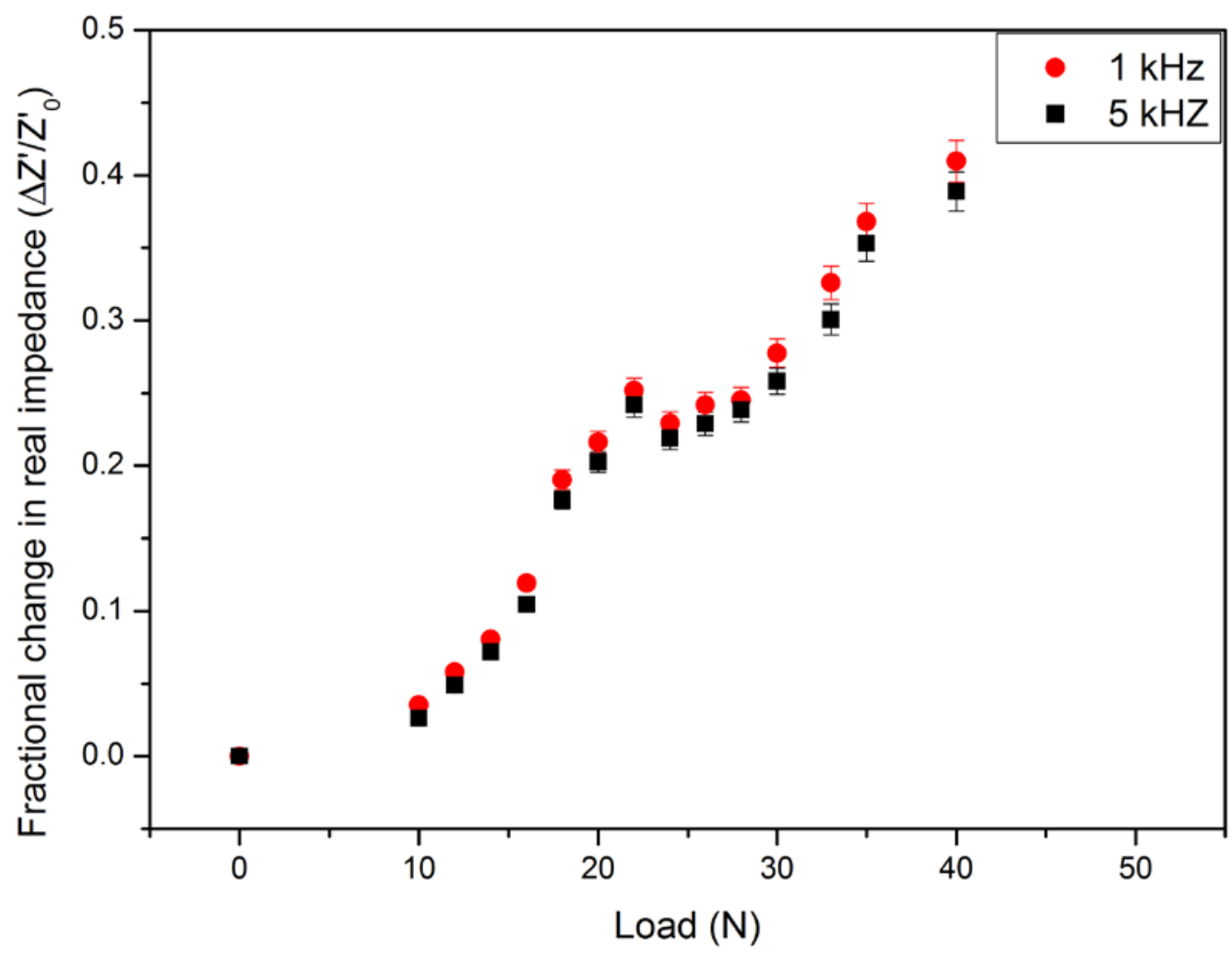

Figure 21: Fractional change in the real impedance versus load for the SIC sensor 


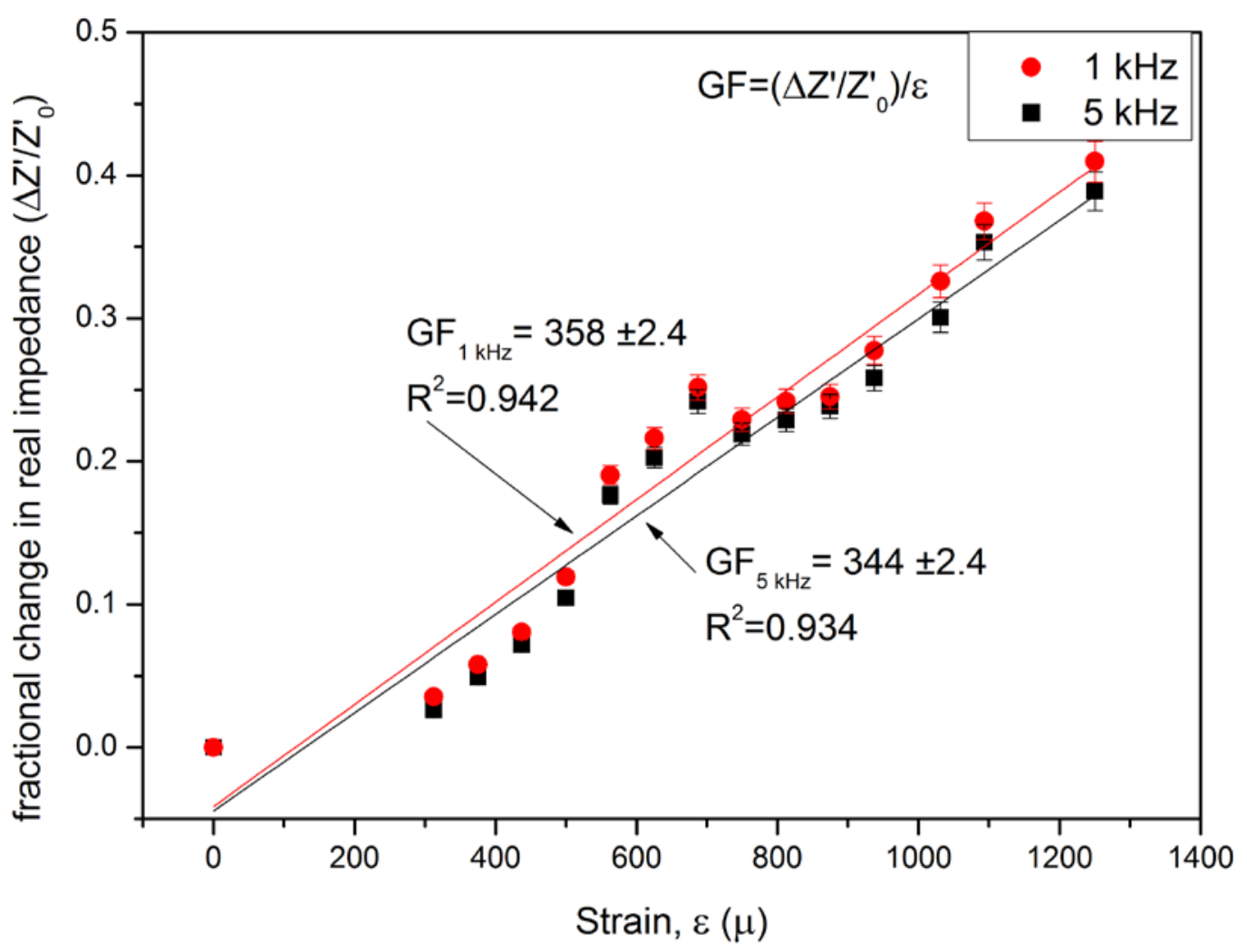

Figure 22: Fractional change in the real impedance versus applied strain for the SIC sensor 